\title{
GEOMETRIC STRUCTURES AND CONFIGURATIONS OF FLAGS IN ORBITS OF REAL FORMS
}

\author{
ELISHA FALBEL, ANTONIN GUILLOUX AND QINGXUE WANG
}

\begin{abstract}
This is an introduction and a survey on geometric structures modelled on closed orbits of real forms acting on spaces of flags. We focus on 3-manifolds and the flag space of all pairs of a point and a line containing it in $\mathbb{P}\left(\mathbb{C}^{3}\right)$. It includes a description of general flag structures which are not necessarily flat and a combinatorial description of flat structures through configurations of flags in closed orbits of real forms. We also review volume and Chern-Simons invariants for those structures.
\end{abstract}

\section{Contents}

1. Introduction

2. Real orbits in flag spaces 2

3. Real flag structures $\quad 5$

4. Bloch group 10

5. Configurations of flags and their invariants 15

6. Representations 20

7. Chern-Simons invariants 25

References $\quad 28$

\section{INTRODUCTION}

Riemannian metrics on 3-manifolds have been studied for a long time. In particular, locally homogeneous metrics are associated to certain classes of manifolds, the most important of them being hyperbolic metrics, that is, those of constant negative curvature. The geometrization conjecture by Thurston, proved by Perelman states that each compact 3-manifold has a decomposition into pieces, glued through tori, such that each of them has a locally homogeneous metric of finite volume defined in its interior.

A natural question is whether 3-manifolds admit other geometric structures in such a way that locally homogeneous structures might describe all of them by an analogous topological decomposition. In this survey we consider one special class of structures, namely, flag structures. They are modeled on the space of flags in $\mathbb{P}\left(\mathbb{C}^{3}\right)$, that is, the space of all pairs formed by a point in $\mathbb{P}\left(\mathbb{C}^{3}\right)$ and a line containing it (see section 2).

The two classical flag structures are path geometry and CR (Cauchy-Riemann) structures. Both geometries were studied by Elie Cartan (see [C24, C]) who constructed adapted fiber bundles and connections whose null curvature examples are locally homogeneous geometric structures. The Riemannian condition of finite volume can be translated in both geometries by a precise description of the structure on the boundary. We review in section 3 a definition of flag structure $([\mathrm{FV}])$ in the

Q. Wang was supported by NSFC grant \#11371092. 
general context of not necessarily flat structures. The advantage of the definition is that it includes both CR and path geometries.

It is not known which manifolds admit flat structures of either path geometry or CR geometry. Both geometries have an underlying contact structure and it is not known yet if there are any constraints on the contact structure in the case a flat flag structure is defined on the manifold.

A locally homogeneous geometry can be described as a $(G, X)$-structure. Here $X$ is a model space where a Lie group $G$ acts transitively. The flat path or CR structures are described as $\left(G_{0}, X_{0}\right)$-structures where $G_{0}$ is a real form of $\operatorname{SL}(3, \mathbb{C})$ and $X_{0}$ is the unique closed orbit of the subgroup $G_{0}$ on the space of flags $\operatorname{SL}(3, \mathbb{C}) / B$, where $B$ is the Borel subgroup of upper triangular matrices (see section 2).

In a more general form we are asking which manifolds are modeled on closed orbits of real forms. In the three dimensional case there are two non-compact real forms $\mathrm{SL}(3, \mathbb{C})$ and $\mathrm{SU}(2,1)$ but both flat geometries can be seen as null curvature models of a flag structure (see [FV] and section 3). More generally, one can prove that all the null curvature models are obtained locally as totally real embeddings into the flag space $\mathrm{SL}(3, \mathbb{C}) / B$ (see $[\mathrm{FV}]$ and section 3 ).

Geometric structures modeled on real forms can be constructed through configurations of flags contained in the closed orbits (see section 6). To each vertex of a simplex of a triangulation we associate a flag and because of the compatibilities given by the gluing maps between the simplices we obtain a representation (the holonomy) of the fundamental group of the manifold into a real form of $\operatorname{PSL}(3, \mathbb{C})$. In some cases one is able to obtain a well defined geometric structure whose holonomy is the holonomy of the representation but this is not always the case. The general case of triangulations decorated with flags in $\mathbb{P}\left(\mathbb{C}^{3}\right)$ was treated in $[\mathrm{BFG}]$ and, in higher dimensions, in [GTZ, GGZ15, DGG16, G]. Decorated triangulations in the case of surfaces were introduced in [FG07] to describe projective structures and the decorated triangulations in the three dimensional case can be seen as a generalisation of this work. Another direct source of decorated triangulations is the work of Thurston constructing hyperbolic 3-manifolds by geometrizing triangulations via ideal hyperbolic tetrahedra (see [T]). The development of effective computations (SnapPea) with hyperbolic manifolds and their invariants gave motivation to understand invariants in this more general case.

In the last section we review some invariants of these decorated triangulations and relate them to the Chern-Simons invariants defined with the help of connections on fiber bundles associated to flag structures. The invariants are also related to K-theory via the third homology of $\operatorname{SL}(3, \mathbb{C})$ and we recall the definition of the Bloch group $\mathcal{B}(F)$ of a field $F$ which describes it in section 4.

Section 5 should be seen as an introduction to the subject of how to obtain an invariant from a decorated triangulation. Here, we aim at invariance with respect to a change in the triangulation. It contains an elementary proof of the triangulation independency of an invariant (defined in the pre-Bloch group) based on Pascal's theorem in the complex projective plane.

We prove (see [FW2] and section 6) that the invariants of flat real flag structures have values in the subgroup of conjugate invariant elements $\mathcal{B}^{+}(\mathbb{C}) \subset \mathcal{B}(\mathbb{C})$ and we give in Proposition 4.8 the relation of this group and $\mathcal{B}(\mathbb{R})$.

We thank C. Gorodski for pointing to us Kostant-Wolff closed orbit theorem 2.3 some years ago and J. M. Veloso for many discussions.

\section{REAL ORBits IN FLAG SPACES}

In this section we describe the models of real flag structures. They are orbits under real forms of complex Lie groups acting on flag spaces. 
2.1. Flag manifolds. A flag manifolds is defined as a quotient $G / P$ where $G$ is a connected complex semisimple Lie group and $P \subset G$ a parabolic subgroup (that is, a connected complex Lie subgroup containing a maximal solvable Lie $\operatorname{subgroup~} B$, also called a Borel subgroup). In this survey we will deal mainly with $G=\operatorname{SL}(n, \mathbb{C})$ and mostly for $n=3$.

The first examples of flag manifolds are given by the following construction. Let $1 \leq d_{1}<\cdots \leq d_{m}<n$ be a sequence of positive integers. Consider the flag manifold of type $\left(d_{1}, \cdots, d_{m}\right)$ :

$$
\operatorname{Flag}_{d_{1} \cdots d_{m}}=\left\{\left(V_{1}, \cdots, V_{m}\right) \mid \operatorname{dim}\left(V_{i}\right)=d_{i} \text { and } V_{1} \subset \cdots \subset V_{m} \subset \mathbb{C}^{n}\right\}
$$

where the inclusion $V_{i} \subset V_{i+1}$ is strict. The group $\operatorname{SL}(n, \mathbb{C})$ acts transitively on this space with isotropy a parabolic group. The case where $\operatorname{dim}\left(V_{i+1}\right)=\operatorname{dim}\left(V_{i}\right)+1$ for all $i$ is called the complete flags space and in this case the parabolic group is the Borel group of all upper triangular matrices in $\operatorname{SL}(n, \mathbb{C})$.

Flag manifolds are compact projective algebraic manifolds and the natural fibration $G / P \rightarrow G / B$ is holomorphic and $G$-equivariant.

Example 2.1. Our most important example is the flag space Flag ${ }_{12}$ of complete flags (that is, lines and planes containing them) in $\mathbb{C}^{3}$. The group $\operatorname{SL}(3, \mathbb{C})$ acts on the space of flags with isotropy $B$, the Borel group of upper triangular matrices. We can describe therefore the space of flags as the homogeneous space Flag $_{12}=$ $\mathrm{SL}(3, \mathbb{C}) / B$. The space of flags is equipped with two projections. One projects the line of a flag into $\mathbb{P}\left(\mathbb{C}^{3}\right)$ on one hand and, on the other, the plane into $\mathbb{P}\left(\mathbb{C}^{3^{*}}\right)$ viewed as a kernel of a linear form.

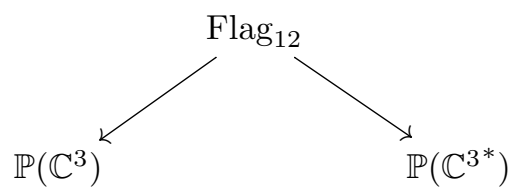

The two projections correspond to the projections into $\mathbb{P}\left(\mathbb{C}^{3}\right)=\operatorname{SL}(3, \mathbb{C}) / P_{1}$ and $\mathbb{P}\left(\mathbb{C}^{3^{*}}\right)=\mathrm{SL}(3, \mathbb{C}) / P_{2}$ where $P_{1}$ and $P_{2}$ are two different parabolic subgroups which fix, respectively, the line and the plane of the flag fixed by the Borel subgroup.

The projections define a complex contact distribution on the tangent space of Flag $_{12}$ generated by the tangent spaces to each of the fibers. We let $\mathfrak{T}^{1}$ and $\mathfrak{T}^{2}$ be the tangent spaces to the fibers.

One can embed Flag $_{12}$ into $\mathbb{P}\left(\mathbb{C}^{3}\right) \times \mathbb{P}\left(\mathbb{C}^{3^{*}}\right)$ as the set of pairs $(z, l) \in \mathbb{P}\left(\mathbb{C}^{3}\right) \times$ $\mathbb{P}\left(\mathbb{C}^{3^{*}}\right)$ satisfying the incidence relation $l(z)=0$.

From the identification

$$
\operatorname{Flag}_{12}=\mathrm{SL}(3, \mathbb{C}) / B \simeq \mathrm{SU}(3) / T^{2},
$$

with $T^{2}$ being the 2 -torus, one obtains the long exact sequence of the fibration $T^{2} \rightarrow \mathrm{SU}(3) \rightarrow$ Flag $_{12}:$

$$
\begin{aligned}
\cdots \pi_{3}\left(T^{2}\right) & \rightarrow \pi_{3}(\mathrm{SU}(3)) \rightarrow \pi_{3}(\mathrm{Flag}) \rightarrow \pi_{2}\left(T^{2}\right) \rightarrow \pi_{2}(\mathrm{SU}(3)) \rightarrow \pi_{2}\left(\mathrm{Flag}_{12}\right) \\
& \rightarrow \pi_{1}\left(T^{2}\right) \rightarrow \pi_{1}(\mathrm{SU}(3)) \rightarrow \pi_{1}(\mathrm{Flag}) \rightarrow \pi_{0}\left(T^{2}\right) \rightarrow \cdots
\end{aligned}
$$

We then observe that $\pi_{1}\left(\mathrm{Flag}_{12}\right)$ is trivial and $\pi_{2}\left(\mathrm{Flag}_{12}\right)=\mathbb{Z} \oplus \mathbb{Z}$. It is also clear that $\pi_{3}\left(\mathrm{Flag}_{12}\right) \simeq \pi_{3}(\mathrm{SU}(3)) \simeq \mathbb{Z}$. Remark that the generators of $\pi_{2}\left(\mathrm{Flag}_{12}\right)$ are given by the fibers of the two canonical projections.

In general, a flag manifold is simply connected and by a theorem of Wang $([\mathrm{Wa}])$ (see also [Ti]) every compact simply connected homogeneous manifold is a torus bundle over a flag space. 
Example 2.2 (The Klein correspondence). In the case $n=4$ there is a classical description of the flag manifolds. In particular, Flag 2 is best described using the Plücker embedding:

$$
p l: \operatorname{Flag}_{2} \rightarrow \mathbb{P}\left(\Lambda^{2}\left(\mathbb{C}^{4}\right)\right)
$$

defined for a plane $\langle Z, W\rangle$ determined by two linearly independent vectors as

$$
p l(\langle Z, W\rangle)=[Z \wedge W] .
$$

Using the basis $e_{i} \wedge e_{j}, i<j$, and corresponding homogeneous coordinates $z^{i j}$, the map $p l$ is an embedding into a quadric in $\mathbb{P}\left(\mathbb{C}^{6}\right)$ (the Klein quadric) defined as

$$
Q_{4}=\left\{\left[z^{i j}\right] \in \mathbb{P}\left(\mathbb{C}^{6}\right) \mid z^{12} z^{34}-z^{13} z^{24}+z^{14} z^{23}=0\right\} .
$$

One can embed Flag ${ }_{123}$ as a degree two variety in $\mathbb{P}\left(\mathbb{C}^{4}\right) \times Q_{4} \times \mathbb{P}\left(\mathbb{C}^{4^{*}}\right)$. Indeed, a point $p=\left[x_{1}, x_{2}, x_{3}, x_{4}\right] \in \mathbb{P}\left(\mathbb{C}^{4}\right)$ is in the plane $m \in Q_{4}$ if and only if $p \wedge m=0$. On the other hand, an hyperplane defined by $l=\left[y_{1}, y_{2}, y_{3}, y_{4}\right] \in \mathbb{P}\left(\mathbb{C}^{4^{*}}\right)$ contains $m$ if and only if the inner product $l\lrcorner m$ is null. This is equivalent to say that $\left(l \wedge e_{i}^{*}\right)(m)=0$ for all $i$.

The flag manifolds correspondences are gathered in the following diagram:

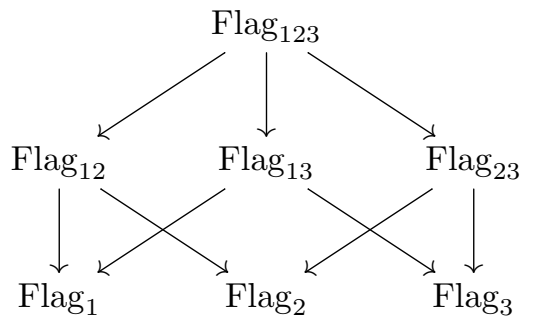

2.2. Real orbits. Let $G_{0}$ be a real form of $G=\mathrm{SL}_{n}(\mathbb{C})$. That is a Lie subgroup with Lie algebra $\mathfrak{g}_{0}$ such that the Lie algebra of $G$ is the complexification $\mathfrak{g}=$ $\mathfrak{g}_{0} \otimes \mathbb{C}$. Denote by $\tau$ the complex conjugation so that $\mathfrak{g}_{0}$ are the fixed points of that involution.

The following is a fundamental description of closed orbits in a flag space under a real form. It is attributed in part to Kostant in [Wol69].

Theorem 2.3 ([Wol69, Theorems 3.3 and 3.6]). Let Flag $=G / P$ be a complex flag space and $G_{0} \subset G$ be a real form.

(1) there exists a unique closed $G_{0}$-orbit $T$ in Flag;

(2) for any $x \in$ Flag, the closed orbit $T$ is contained in the closure of $G_{0} x$ and

$$
\operatorname{dim}_{\mathbb{R}} G_{0} x \geq \operatorname{dim}_{\mathbb{R}} T \geq \operatorname{dim}_{\mathbb{C}} \text { Flag }
$$

with equality if and only Flag is a variety defined over $\mathbb{R}$. In this case $T$ is the set of real points of the associated real flag variety.

Example 2.4. The first example is that of $\operatorname{SL}(2, \mathbb{C})$ acting on $\mathbb{P}^{1}(\mathbb{C})$. There are two real forms up to equivalence. The compact form $\mathrm{SU}(2)$ acts transitively on the flag space, as is always the case for any compact real form $([\mathrm{Ti}])$. For $G_{0}=\mathrm{SL}(2, \mathbb{R})$ the unique closed orbit is $\mathbb{P}(\mathbb{R})$.

Example 2.5. Consider $\mathrm{SL}(3, \mathbb{C})$ acting on $\mathbb{P}^{2}(\mathbb{C})$. There are three real forms, the non-compact ones being $\mathrm{SL}(3, \mathbb{R})$ and $\mathrm{SU}(2,1)$ (matrices preserving a hermitian form with two positive and one negative eigenvalues). The closed orbit of $\operatorname{SL}(3, \mathbb{R})$ is $\mathbb{P}\left(\mathbb{R}^{3}\right)$ and geometric structures on surfaces modeled on this orbit are called projective structures. On the other hand, consider the group $\mathrm{SU}(2,1) \subset \mathrm{SL}(3, \mathbb{C})$ 
preserving the Hermitian form $\langle z, w\rangle=w^{*} J z$ defined on $\mathbb{C}^{3}$ by the matrix

$$
J=\left(\begin{array}{lll}
0 & 0 & 1 \\
0 & 1 & 0 \\
1 & 0 & 0
\end{array}\right)
$$

and the following cones in $\mathbb{C}^{3}$ :

$$
\begin{gathered}
V_{0}=\left\{z \in \mathbb{C}^{3}-\{0\} \quad: \quad\langle z, z\rangle=0\right\}, \\
V_{-}=\left\{z \in \mathbb{C}^{3}:\langle z, z\rangle<0\right\} .
\end{gathered}
$$

Let $\pi: \mathbb{C}^{3} \backslash\{0\} \rightarrow \mathbb{P}\left(\mathbb{C}^{3}\right)$ be the canonical projection. Then $\mathbb{H}_{\mathbb{C}}^{2}=\pi\left(V_{-}\right)$is the complex hyperbolic space and its boundary is

$$
\mathbb{S}^{3}=\pi\left(V_{0}\right)=\left\{\left.[x, y, z] \in \mathbb{C P}^{2}|x \bar{z}+| y\right|^{2}+z \bar{x}=0\right\} .
$$

is the closed orbit. The group of biholomorphic transformations of $\mathbb{H}_{\mathbb{C}}^{2}$ is then $\mathrm{PU}(2,1)$, the projectivization of $\mathrm{SU}(2,1)$. Three-dimensional manifolds with geometric structures modeled on the the closed orbit $\mathbb{S}^{3}$ are called $\mathrm{CR}$ structures.

Example 2.6. Consider $\mathrm{SL}(3, \mathbb{C})$ acting on $\operatorname{Flag}_{12}\left(\mathbb{C}^{3}\right)$. Again, the real forms are $\mathrm{SL}(3, \mathbb{R})$ and $\mathrm{SU}(2,1)$. The closed orbit by the action of $\mathrm{SL}(3, \mathbb{R})$ is the set of real flags consisting of one point in $\mathbb{P}\left(\mathbb{R}^{3}\right)$ and a line in $\mathbb{P}\left(\mathbb{R}^{3}\right)$ passing through that point.

An element $x \in \mathbb{S}^{3} \in \mathbb{P}\left(\mathbb{C}^{3}\right)$ gives rise to a flag in $\operatorname{Flag}_{12}\left(\mathbb{C}^{3}\right)$ where the line corresponds to the unique complex line tangent to $\mathbb{S}^{3}$ at $x$. More explicitly, the map

$$
\begin{aligned}
\phi_{C R}: \mathbb{S}^{3} & \rightarrow \operatorname{Flag}_{12}\left(\mathbb{C}^{3}\right) \\
x & \mapsto(x,\langle., x\rangle)
\end{aligned}
$$

is an equivariant diffeomorphism of $\mathbb{S}^{3}$ with the closed orbit.

\section{REAL FLAG STRUCTURES}

This section is a review of $[\mathrm{FV}]$. We suppose $M$ is a real three dimensional manifold in the following. Real flag structures are geometric structures on real manifolds which are generalizations of locally homogeneous geometric structures modelled on orbits of real forms in a flag space. They are not locally homogeneous in general and they are analogues of riemannian manifolds viewed as an abstraction of submanifolds of euclidean space.

We restrict ourselves to geometric structures related to the flag space Flag ${ }_{12}$ as defined in $[\mathrm{FV}]$. In the next subsection we identify the structure using totally real embeddings in the flag space.

3.1. Real submanifolds in flag space. Let $\phi: M \rightarrow \mathrm{SL}(3, \mathbb{C}) / B$ be an embedding and $\phi_{*}: T M \rightarrow T \mathrm{SL}(3, \mathbb{C}) / B$ be its differential. Extend this map to the complexification $T M^{\mathbb{C}}$ of $T M$ defining $\phi_{*}: T M^{\mathbb{C}} \rightarrow T \mathrm{SL}(3, \mathbb{C}) / B$ by $\phi_{*}(i v)=J \phi_{*}(v)$, where $J$ is the complex structure on the tangent space of the complex manifold $\mathrm{SL}(3, \mathbb{C}) / B$.

Definition 3.1. An embedding $\phi: M \rightarrow \mathrm{SL}(3, \mathbb{C}) / B$ is totally real if, for every $p \in M$,

$$
\phi_{*}: T_{p} M^{\mathbb{C}} \rightarrow T_{p} S L(3, \mathbb{C}) / B
$$

is an isomorphism. 
One defines two complex one dimensional distributions $T^{1}, T^{2} \subset T M^{\mathbb{C}}$ which correspond to $\mathfrak{T}^{1}$ and $\mathfrak{T}^{2}$ on the flag space $\mathrm{SL}(3, \mathbb{C}) / B$ (see example 2.1 ).

A flag structure on a real 3-manifold $M$ is a generalization of this definition to the case there is no known real immersion into the flag space. The locally homogeneous examples arise naturally from closed orbits in the flag space by the action of a non-compact real form of $\mathrm{SL}(3, \mathbb{C})$.

3.1.1. Spherical $C R$ geometry and $\mathrm{SU}(2,1)$. Spherical CR geometry is modeled on the sphere $\mathbb{S}^{3}$ equipped with a natural $\mathrm{PU}(2,1)$ action as in Example 2.5. Another approach to the CR structure on the sphere is to use the map $\phi_{C R}: \mathbb{S}^{3} \rightarrow$ Flag $_{12}$ defined in Example 2.6 (that is $x \mapsto(x,\langle., x\rangle)$ ) as a totally real embedding and then define a flag structure as above.

3.1.2. Real path geometry and $\mathrm{SL}(3, \mathbb{R})$. Flat path geometry is the geometry of real flags in $\mathbb{R}^{3}$. That is the geometry of the space of all couples $[p, l]$ where $p \in \mathbb{R} P^{2}$ and $l$ is a real projective line containing $p$. The space of flags is identified to the quotient

$$
\mathrm{SL}(3, \mathbb{R}) / B_{\mathbb{R}}
$$

where $B_{\mathbb{R}}$ is the Borel group of all real upper triangular matrices. The inclusion

$$
\phi_{\mathbb{R}}: \operatorname{SL}(3, \mathbb{R}) / B_{\mathbb{R}} \rightarrow \mathrm{SL}(3, \mathbb{C}) / B
$$

is clearly a totally real embedding.

\subsection{Flag structures in dimension 3.}

Definition 3.2. A flag structure on $M$ is a choice of two one dimensional subbundles $T^{1}$ and $T^{2}$ in $T^{\mathbb{C}}$ such that $T^{1} \cap T^{2}=\{0\}$ and such that $T^{1} \oplus T^{2}$ is a contact distribution.

The condition that $T^{1} \oplus T^{2}$ be a contact distribution means that, locally, there exists a one form $\theta \in T^{*} M \otimes \mathbb{C}$ such that $\operatorname{ker} \theta=T^{1} \oplus T^{2}$ and $d \theta \wedge \theta$ is never zero.

This definition contains two special cases, namely,

- CR structures, which arise when $T^{2}=\overline{T^{1}}$.

- Path geometries, which are defined when $T^{1}$ and $T^{2}$ are complexifications of real one dimensional sub-bundles of $T M$.

Path geometries ([C24] and section 8.6 of [IL]) and CR structures ([C], see also $[\mathrm{J}])$ in three dimensional manifolds were studied by Cartan. He defined what is called today a Cartan connection (see 3.3) for those two structures. The CR case was generalized to higher dimensions in [Ta, CM]. The general definition interpolates between the two geometries.

3.3. Connection forms and curvatures. In Riemannian geometry, the orthogonal coframe bundle is a geometric object which allows to define a connection and curvature. More generally, a homogeneous space $G / H$ where $H \subset G$ is a Lie subgroup might be used as a flat model space for a geometry on a manifold $M$ which is encoded in a $H$-principal bundle

$$
Y \rightarrow M \text {. }
$$

Recall that the fundamental vertical fields on $Y$ are defined by $X^{*}(y)=\frac{d}{d t}{ }_{t=0} y e^{t X}$ where $e^{t X}$ is the one parameter group generated by $X \in \mathfrak{h}$. In Cartan's idea, the bundle $Y$ should be viewed as a non-flat analogue of the $H$-principal bundle $G \rightarrow$ $G / H$. A Cartan connection $\pi$ is then a $\mathfrak{g}$-valued 1 -form defined on $Y$ which imitates the Maurer-Cartan forms. More precisely the connection satisfies $\pi\left(X^{*}\right)=X$ for all $X \in \mathfrak{h}$, and is an isomorphism of vector spaces at each point. Moreover, it has the 
same invariance property (with respect to the subgroup $H$ ) as the Maurer-Cartan forms on the group $G$, that is

$$
R_{h}{ }^{*} \pi=A d\left(h^{-1}\right) \pi,
$$

where $R_{h}(g)=g . h$ is right multiplication by $h$ and $A d\left(h^{-1}\right)$ is the adjoint by $h^{-1}$ defined on the Lie algebra $\mathfrak{g}$.

The curvature of a Cartan connection is given by

$$
\Pi=d \pi+\pi \wedge \pi \text {. }
$$

The fundamental observation related to this construction is that, if the curvature is null, then the bundle $Y$ is obtained locally through a local map from $M$ to $G / H$ by pulling-back the bundle $G \rightarrow G / H$.

Cartan analyzed several geometries, among them conformal and projective geometries before considering CR structures and path geometry over three manifolds. He defined a Cartan connection for each of those geometries on an appropriate bundle. This appropriate bundle is not always a coframe bundle but in several cases it is a coframe bundle over a natural bundle over the manifold. It corresponds to certain natural fiber bundles over the homogeneous space model.

\subsubsection{The CR Cartan connection. Define}

$$
\mathrm{SU}(2,1)=\left\{g \in \mathrm{SL}(3, \mathbb{C}) \mid \bar{g}^{T} Q g=Q\right\}
$$

with Hermitian form $Q$ given by

$$
Q=\left(\begin{array}{ccc}
0 & 0 & i / 2 \\
0 & 1 & 0 \\
-i / 2 & 0 & 0
\end{array}\right)
$$

The group $\mathrm{SU}(2,1)$ acts on $\mathbb{C}^{3}$ on the left preserving the cone

$$
\left\{z \in \mathbb{C}^{3} \mid \bar{z}^{T} Q z=0\right\} .
$$

The projectivization of this cone is $S^{3} \subset \mathbb{P}^{3}$ as in 2.5.

The elements of the Lie algebra $\mathfrak{s} u(2,1)$ are represented by the matrices

$$
\left(\begin{array}{ccc}
u & -2 i \bar{y} & w \\
x & a & y \\
z & 2 i \bar{x} & -\bar{u}
\end{array}\right)
$$

where ia $\in \mathbb{R}, z, w \in \mathbb{R}, x, y \in \mathbb{C}, u \in \mathbb{C}$ and $u-\bar{u}=-a$. Observe that the Lie algebra $\mathfrak{g}=\mathfrak{s} u(2,1)$ is graded:

$$
\mathfrak{g}=\mathfrak{g}^{-2} \oplus \mathfrak{g}^{-1} \oplus \mathfrak{g}^{0} \oplus \mathfrak{g}^{1} \oplus \mathfrak{g}^{2}
$$

where

$$
\begin{gathered}
\mathfrak{g}^{-2}=\left\{\left(\begin{array}{lll}
0 & 0 & 0 \\
0 & 0 & 0 \\
z & 0 & 0
\end{array}\right)\right\} \mathfrak{g}^{-1}=\left\{\left(\begin{array}{ccc}
0 & 0 & 0 \\
x & 0 & 0 \\
0 & 2 i \bar{x} & 0
\end{array}\right)\right\} \\
\mathfrak{g}^{0}=\left\{\left(\begin{array}{ccc}
u & 0 & 0 \\
0 & a & 0 \\
0 & 0 & -\bar{u}
\end{array}\right)\right\} \quad \mathfrak{g}^{1}=\left\{\left(\begin{array}{ccc}
0 & -2 i \bar{y} & 0 \\
0 & 0 & y \\
0 & 0 & 0
\end{array}\right)\right\} \quad \mathfrak{g}^{2}=\left\{\left(\begin{array}{lll}
0 & 0 & w \\
0 & 0 & 0 \\
0 & 0 & 0
\end{array}\right)\right\}
\end{gathered}
$$

Observe that

where

$$
\mathfrak{g}^{0}=\mathbb{R} \oplus \mathfrak{u}(1),
$$

$$
\mathfrak{u}(1)=\left\{\left(\begin{array}{ccc}
-i q / 2 & 0 & 0 \\
0 & i q & 0 \\
0 & 0 & -i q / 2
\end{array}\right)\right\}
$$


with $q \in \mathbb{R}$ and

$$
\mathbb{R}=\left\{\left(\begin{array}{ccc}
r & 0 & 0 \\
0 & 0 & 0 \\
0 & 0 & -r
\end{array}\right)\right\}
$$

with $r \in \mathbb{R}$.

Define the subalgebras

$$
\begin{gathered}
\mathfrak{h}=\mathfrak{g}^{0} \oplus \mathfrak{g}^{1} \oplus \mathfrak{g}^{2} \quad \mathfrak{a}=\mathbb{R} \oplus \mathfrak{g}^{1} \oplus \mathfrak{g}^{2} \\
\mathfrak{b}=\mathfrak{u}(1) \oplus \mathfrak{g}^{1} \oplus \mathfrak{g}^{2} \quad \mathfrak{n}=\mathfrak{g}^{1} \oplus \mathfrak{g}^{2}
\end{gathered}
$$

The isotropy of the action of $S U(2,1) / K$ on $S^{3}$ at the point $[1,0,0]^{T}$ is the group $H=C U(1) \ltimes N$ (whose Lie algebra is $\mathfrak{h}$ ), represented (up to $K$ ) by matrices of the form

$$
\left(\begin{array}{ccc}
a & -2 i \bar{a} \bar{b} & a(s-i b \bar{b}) \\
0 & \frac{\bar{a}}{a} & b \\
0 & 0 & \bar{a}^{-1}
\end{array}\right)
$$

where $s \in \mathbb{R}, b \in \mathbb{C}$. $N$ is the Heisenberg group represented by matrices

$$
\left(\begin{array}{ccc}
1 & -2 i \bar{b} & s-i b \bar{b} \\
0 & 1 & b \\
0 & 0 & 1
\end{array}\right)
$$

Define the following subgroups of $H ; A=\mathbb{R}^{+} \ltimes N$ (whose Lie algebra is $\mathfrak{a}$ ), $B=U(1) \ltimes N($ whose Lie algebra is $\mathfrak{b})$ and $N$ (whose Lie algebra is $\mathfrak{n}$ ). The inclusions between these groups, and their corresponding quotient spaces, can be visualized in the following diagrams
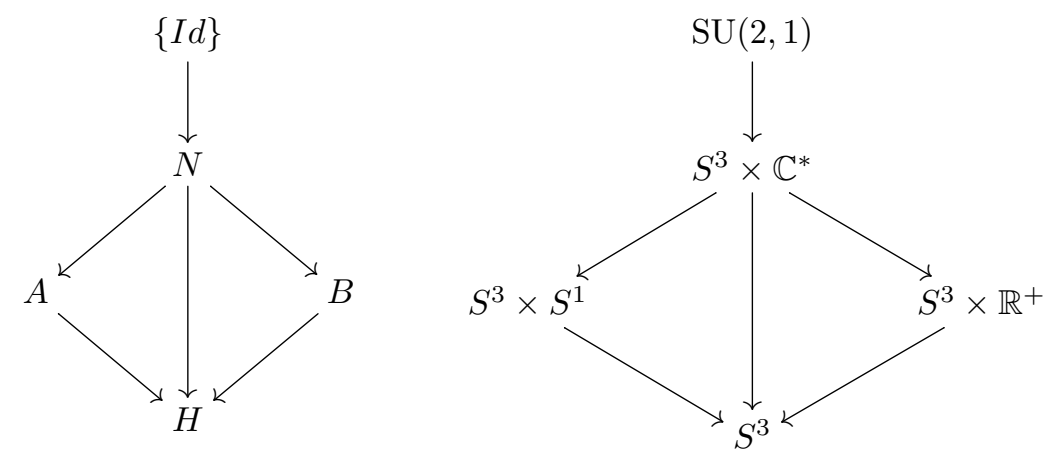

A geometrical interpretation of $S U(2,1) / N$ is obtained considering the canonical bundle of the sphere, that is the restriction of $\Lambda^{2} \mathbb{C}^{2}$ to the sphere, and deleting from it the zero section. This is a trivial $\mathbb{C}^{*}$ bundle over the sphere (it has a non-zero section given by the restriction of $d z^{1} \wedge d z^{2}$ to the sphere). We obtain $S U(2,1) / N \simeq S^{3} \times \mathbb{C}^{*}, S U(2,1) / A \simeq S^{3} \times S^{1}$ and $S U(2,1) / B \simeq S^{3} \times \mathbb{R}^{+}$.

The construction of the appropriate $H$-principal bundle $Y$ over a CR manifold $M$, follows the diagram above. Although $Y$ isn't a coframe bundle over $M$ it can be obtained as a coframe bundle over an intermediate $\mathbb{R}^{+}$-bundle over a $M$. This is a particular instance of Cartan's technique of prolongation. 


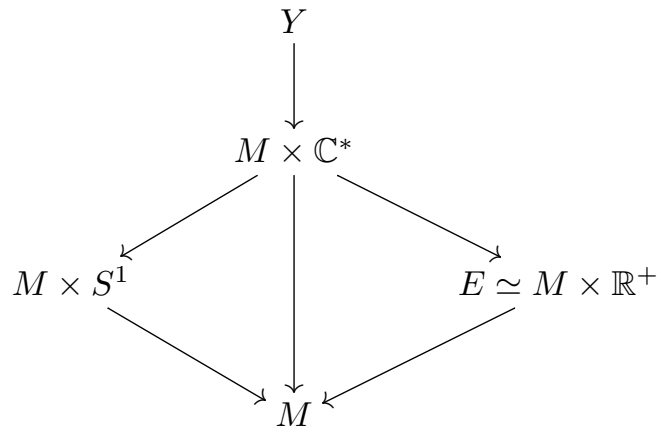

We will not describe here the construction of $Y$ and it is Cartan connection (see $[\mathrm{J}]$ ). However, the bundle $E$ isomorphic to $M \times \mathbb{R}^{+}$which depends only on the existence of a co-oriented contact structure is constructed easily. Each contact plane on $M$ is given locally by 1 -forms such that, in different neighbourhoods, differ by a positive function. One considers then the $\mathbb{R}^{+}$-bundle $E$ of those contact forms. The bundle $Y \rightarrow E$ is a $B$-bundle and on the bundle $Y \rightarrow M$ there exists a Cartan connection.

Proposition 3.3 (Cartan, $[\mathrm{C}]$ ). On the $H$-bundle $Y \rightarrow M$ there exists a 1-form $\pi: T Y \rightarrow s u(2,1)$ satisfying:

1. $\pi_{p}: T_{p} Y \rightarrow s u(2,1)$ is an isomorphism

2. If $X \in \mathfrak{h}$ and $X^{*} \in T Y$ is the vertical vector field canonically associated to $X$ then $\pi\left(X^{*}\right)=X$.

3. If $h \in H$ then $\left(R_{h}\right)^{*} \pi=A d_{h^{-1}} \pi$

3.3.2. The flag structure connection. The Lie algebra of $\mathrm{SL}(3, \mathbb{C})$ decomposes in the following direct sum of vector subspaces:

$$
\mathfrak{s l}(3, \mathbb{C})=\mathfrak{g}^{-2} \oplus \mathfrak{g}^{-1} \oplus \mathfrak{g}^{0} \oplus \mathfrak{g}^{1} \oplus \mathfrak{g}^{2},
$$

where

$$
\begin{gathered}
\mathfrak{g}^{-2}=\left\{\left(\begin{array}{lll}
0 & 0 & 0 \\
0 & 0 & 0 \\
z & 0 & 0
\end{array}\right)\right\}, \quad \mathfrak{g}^{-1}=\left\{\left(\begin{array}{lll}
0 & 0 & 0 \\
x & 0 & 0 \\
0 & y & 0
\end{array}\right)\right\}, \\
\mathfrak{g}^{0}=\left\{\left(\begin{array}{lll}
u & 0 & 0 \\
0 & v & 0 \\
0 & 0 & w
\end{array}\right)\right\} \quad \text { with } u+v+w=0, \\
\mathfrak{g}^{1}=\left\{\left(\begin{array}{lll}
0 & x & 0 \\
0 & 0 & y \\
0 & 0 & 0
\end{array}\right)\right\}, \quad \mathfrak{g}^{2}=\left\{\left(\begin{array}{lll}
0 & 0 & z \\
0 & 0 & 0 \\
0 & 0 & 0
\end{array}\right)\right\} .
\end{gathered}
$$

One obtains the graded decomposition of $\mathfrak{s l}(3, \mathbb{C})$, where $\mathfrak{b}=\mathfrak{g}^{0} \oplus \mathfrak{g}^{1} \oplus \mathfrak{g}^{2}$ is the space of upper triangular matrices with null trace. The tangent space of $\mathrm{SL}(3, \mathbb{C}) / B$ at $[B]$ is identified to

$$
\mathfrak{s l}(3, \mathbb{C}) / \mathfrak{b}=\mathfrak{g}^{-2} \oplus \mathfrak{g}^{-1} .
$$

The choice of the reference flag whose isotropy is $B$ defines a decomposition of $\mathfrak{g}^{-1}=\mathfrak{t}_{1} \oplus \mathfrak{t}_{2}$, with $\mathfrak{g}^{-2}=\left[\mathfrak{t}_{1}, \mathfrak{t}_{2}\right]$, corresponding to the two parabolic subgroups with Lie algebras $\mathfrak{p}_{1}=\mathfrak{t}_{1} \oplus \mathfrak{g}^{0} \oplus \mathfrak{g}^{1} \oplus \mathfrak{g}^{2}$ and $\mathfrak{p}_{2}=\mathfrak{t}_{2} \oplus \mathfrak{g}^{0} \oplus \mathfrak{g}^{1} \oplus \mathfrak{g}^{2}$.

One propagates the decomposition of the tangent space at $[B]$ to the whole flag space by the action of $\operatorname{SL}(3, \mathbb{C})$ to obtain the two fields of complex vectors $\mathfrak{T}^{1}$ and $\mathfrak{T}^{2}$.

We proceed to obtain a $\mathbb{C}^{*}$-bundle $E$ over a real three manifold equipped with a flag structure. We consider the forms $\theta$ on $T^{\mathbb{C}}$ such that $\operatorname{ker} \theta=T^{1} \oplus T^{2}$. Define $E$ to be the $\mathbb{C}^{*}$-bundle of all such forms. This bundle is trivial if and only if there exists a globally defined non-vanishing form $\theta$. 
As in the case of $\mathrm{PU}(2,1)$ and $\operatorname{SL}(3, \mathbb{R})$ one can obtain a frame bundle $Y$ over $E$. The bundle $Y$ can also be fibered over the manifold $M$. The bundle $Y \rightarrow M$ is a principal bundle with structure group $H=B / \mathbb{C}^{*}$ where $B$ is the Borel group of upper triangular matrices.

Observe that, although $M$ is a real manifold, each fiber is a complex space of dimension five which can be identified to the Borel subgroup $H$. The real dimension of $Y$ is 13 which is $\operatorname{dim}_{\mathbb{R}} \mathrm{SL}(3, \mathbb{C})-3$. This dimension difference does not allow us to obtain a genuine Cartan connection but a slight generalization of the definition.

Proposition $3.4([\mathrm{FV}])$. There exists on $Y$ a 1-form $\pi: T Y \rightarrow \mathfrak{s l}(3, \mathbb{C})$ satisfying: 0. $\pi_{p}: T_{p} Y \rightarrow \mathfrak{s l}(3, \mathbb{C})$ is injective.

1. If $X \in \mathfrak{h}$ and $X^{*} \in T Y$ is the vertical vector field canonically associated to $X$ then $\pi\left(X^{*}\right)=X$.

2. If $h \in H$ then $\left(R_{h}\right)^{*} \pi=A d_{h^{-1}} \pi$

Note that contrary to the usual definition of Cartan connection we don't impose that $\pi_{p}: T_{p} Y \rightarrow \mathfrak{s l}(3, \mathbb{C})$ be an isomorphism as the dimensions are different.

A local characterization of null curvature flag geometry is given in the following theorem. Recall definition 3.1 of a totally real embedding $\phi: M \rightarrow \operatorname{SL}(3, \mathbb{C}) / B$ and its associated flag structure which correspond to $\phi_{*}\left(T^{1}\right)=\mathfrak{T}_{1}$ and $\phi_{*}\left(T^{2}\right)=\mathfrak{T}_{2}$ on the flag space $\mathrm{SL}(3, \mathbb{C}) / B$

Theorem 3.5 ([FV]). A totally real immersion $\phi: N \rightarrow \mathrm{SL}(3, \mathbb{C}) / B$ with induced flag structure on $T M^{\mathbb{C}}$ given by $T^{1}$ and $T^{2}$ as above has adapted connection having null curvature. Conversely a flag structure whose adapted connection has zero curvature is obtained locally through a totally real embedding as above.

Observe that null curvature does not define a unique flag structure on a real manifold but instead decides whether is can be embedded as a totally real submanifold in flag space.

One can then define the holonomy of a flat flag structure. Indeed, for any loop starting at a point $p \in M$ one can follow the immersion of $M$ along the path and obtain an element of $\operatorname{PSL}(3, \mathbb{C})$ which will translate the original neighbourhood to the final embedding of it.

\section{BLOCH GROUP}

In this section, we will review the (pre-)Bloch group of a field $F$, the Rogers's dilogarithm function and the relations with Algebraic K-theory. We will mainly discuss the cases for $F=\mathbb{R}$ or $F=\mathbb{C}$.

\subsection{Basic definitions and properties.}

Definition 4.1. Let $F$ be a field. Let $\mathbb{Z}[F \backslash\{0,1\}]$ be the free abelian group with generators $[x], x \in F \backslash\{0,1\}$. A 5-term relation is an element of $\mathbb{Z}[F \backslash\{0,1\}]$ with the following form:

$$
T(x, y):=[x]-[y]+\left[\frac{y}{x}\right]-\left[\frac{1-x^{-1}}{1-y^{-1}}\right]+\left[\frac{1-x}{1-y}\right], x \neq y \in F \backslash\{0,1\} .
$$

Let $\mathcal{T}$ be the subgroup of $\mathbb{Z}[F \backslash\{0,1\}]$ generated by all the 5 -term relations $T(x, y), x \neq y \in F \backslash\{0,1\}$. We define the pre-Bloch group $\mathcal{P}(F)$ of $F$ to be the quotient group $\mathbb{Z}[F \backslash\{0,1\}] / \mathcal{T}$.

Next we consider the tensor product $F^{*} \otimes_{\mathbb{Z}} F^{*}$, where $F^{*}$ is the multiplicative group of $F$. Let $A S$ be the subgroup of $F^{*} \otimes_{\mathbb{Z}} F^{*}$ generated by the subset $\left\{x \otimes y+y \otimes x \mid x, y \in F^{*}\right\}$. We define $\bigwedge^{2} F^{*}:=\left(F^{*} \otimes_{\mathbb{Z}} F^{*}\right) / A S$. For $x, y \in F^{*}$, we 
will denote by $x \wedge y$ the image of $x \otimes y$ in $\wedge^{2} F^{*}$.

Define a homomorphism $\lambda_{0}: \mathbb{Z}[F \backslash\{0,1\}] \rightarrow \bigwedge^{2} F^{*}$ as follows: for each generator $[z], z \in F \backslash\{0,1\}, \lambda_{0}([z])=(1-z) \wedge z$. Then we can check that $\lambda_{0}(T(x, y))=0$. Hence $\lambda_{0}$ induces a homomorphism

$$
\lambda: \mathcal{P}(F) \rightarrow \bigwedge^{2} F^{*} .
$$

Definition 4.2. The Bloch group $\mathcal{B}(F)$ of $F$ is the kernel of the homomorphism $\lambda$. It is a subgroup of $\mathcal{P}(F)$.

In this paper we will mainly consider the cases $F=\mathbb{R}$ or $F=\mathbb{C}$. When $F=\mathbb{C}$, by [Sah, Sus], both $\mathcal{P}(\mathbb{C})$ and $\mathcal{B}(\mathbb{C})$ are uniquely divisible groups, i.e. they are $\mathbb{Q}$-vector spaces. In particular they are torsion-free. On the other hand, if $F=\mathbb{R}$, then there exists non-trivial torsion. For example, for all $x \in \mathbb{R}-\{0,1\}$, the element $[x]+[1-x] \in \mathcal{B}(\mathbb{R})$ does not depend on $x$ and has order six ([Sus]).

The complex conjugation gives rise to a natural involution:

$$
\tau: \mathbb{Z}[\mathbb{C} \backslash\{0,1\}] \rightarrow \mathbb{Z}[\mathbb{C} \backslash\{0,1\}] .
$$

As $\tau$ preserves the 5-term relation (4.1.1), it induces an involution on the pre-Bloch group $\mathcal{P}(\mathbb{C})$ which we will also denote by $\tau$. Set

$$
\mathcal{P}(\mathbb{C})^{+}=\{x \in \mathcal{P}(\mathbb{C}) \mid \tau(x)=x\}
$$

and

$$
\mathcal{P}(\mathbb{C})^{-}=\{x \in \mathcal{P}(\mathbb{C}) \mid \tau(x)=-x\} .
$$

They are the corresponding \pm 1 -eigenspaces of $\tau$. Then we see

$$
\mathcal{P}(\mathbb{C})=\mathcal{P}(\mathbb{C})^{+} \oplus \mathcal{P}(\mathbb{C})^{-} .
$$

Analogously,

$$
\mathcal{B}(\mathbb{C})=\mathcal{B}(\mathbb{C})^{+}+\mathcal{B}(\mathbb{C})^{-},
$$

where $\mathcal{B}(\mathbb{C})^{+}=\{x \in \mathcal{B}(\mathbb{C}) \mid \tau(x)=x\}$ and $\mathcal{B}(\mathbb{C})^{-}=\{x \in \mathcal{B}(\mathbb{C}) \mid \tau(x)=-x\}$. Clearly $\mathcal{B}(\mathbb{C})^{+}\left(\right.$resp. $\left.\mathcal{B}(\mathbb{C})^{-}\right)$is a subgroup of $\mathcal{P}(\mathbb{C})^{+}\left(\right.$resp. $\left.\mathcal{P}(\mathbb{C})^{-}\right)$.

Definition 4.3. The Bloch-Wigner function is defined as follows,

$$
D(x)=\arg (1-x) \log |x|-\operatorname{Im}\left(\int_{0}^{x} \log (1-t) \frac{d t}{t}\right), \forall x \in \mathbb{C} \backslash\{0,1\} .
$$

It is well-defined and real analytic on $\mathbb{C}-\{0,1\}$ and extends to a continuous function on $\mathbb{C} P^{1}$ by defining $D(0)=D(1)=D(\infty)=0$. It is well-known that it satisfies the 5-term relation (4.1.1). Hence it gives rise to a well-defined homomorphism:

$$
D: \mathcal{P}(\mathbb{C}) \rightarrow \mathbb{R}
$$

given by

$$
D\left(\sum_{i=1}^{k} n_{i}\left[x_{i}\right]\right)=\sum_{i=1}^{k} n_{i} D\left(x_{i}\right)
$$

Since $D(\bar{z})=-D(z)$ for any $z \in \mathbb{C}$, we obtain that

$$
D(u)=0, \forall u \in \mathcal{P}(\mathbb{C})^{+} .
$$

For the proofs of the above-mentioned properties of the dilogarithm function $D$, see [B1] Lecture 6. 
4.2. Rogers's dilogarithm. This function was introduced and studied in [Ro]. Here we use a slightly modified version (See [PS, Sus, Lic]).

Definition 4.4. The Rogers's dilogarithm is defined as follows:

$$
L(x)=\sum_{n=1}^{\infty} \frac{x^{n}}{n^{2}}+\frac{1}{2} \log x \log (1-x)-\frac{\pi^{2}}{6}, 0<x<1 .
$$

Note it is differentiable with the derivative

$$
L^{\prime}(x)=-\frac{1}{2}\left\{\frac{\log (1-x)}{x}+\frac{\log (x)}{1-x}\right\}, 0<x<1 .
$$

By continuity, we have

$$
L(1)=0, \quad L(0)=-\frac{\pi^{2}}{6} .
$$

It is known that (see [Ro, Kir, Za])

$$
L\left(\frac{\sqrt{5}-1}{2}\right)=\frac{\pi^{2}}{10}-\frac{\pi^{2}}{6}=-\frac{\pi^{2}}{15}
$$

and

$$
L\left(\left(\frac{\sqrt{5}-1}{2}\right)^{2}\right)=L\left(\frac{3-\sqrt{5}}{2}\right)=\frac{\pi^{2}}{15}-\frac{\pi^{2}}{6}=-\frac{\pi^{2}}{10} .
$$

By [Sus], we have the group homomorphism $r: \mathcal{P}(\mathbb{R}) \rightarrow \mathbb{R} / \pi^{2} \mathbb{Z}$, defined as follows: for each generator $[x], x \in \mathbb{R} \backslash\{0,1\}$,

$$
r([x])= \begin{cases}L(x) \bmod \pi^{2} \mathbb{Z}, & \text { if } 0<x<1 \\ -L\left(\frac{1}{x}\right) \bmod \pi^{2} \mathbb{Z}, & \text { if } x>1 \\ \left(-\frac{\pi^{2}}{6}+L\left(\frac{1}{1-x}\right)\right) \bmod \pi^{2} \mathbb{Z}, & \text { if } x<0\end{cases}
$$

It was proved in [FS] that on the torsion part of $\mathcal{B}(\mathbb{R}), r$ is injective. It is conjectured that $r$ is injective on the whole $\mathcal{B}(\mathbb{R})$.

Example 4.5. Let $u=\left(\frac{\sqrt{5}-1}{2}\right)^{2}$ and $v=\left(\frac{\sqrt{5}+1}{2}\right)^{2}$. We can check that both $[u]$ and $[v]$ belong to $\mathcal{B}(\mathbb{R})$, i.e. $\lambda([u])=\lambda([v])=0$. Note $u v=1$. By (4.2.2) and (4.2.3), we have

$$
r([u])=L(u) \bmod \pi^{2} \mathbb{Z}=-\frac{\pi^{2}}{10} \bmod \pi^{2} \mathbb{Z}
$$

and

$$
r([v])=-L\left(\frac{1}{v}\right) \bmod \pi^{2} \mathbb{Z}=-L(u) \bmod \pi^{2} \mathbb{Z}=\frac{\pi^{2}}{10} \bmod \pi^{2} \mathbb{Z} .
$$

Since $r$ is injective on the torsion part of $\mathcal{B}(\mathbb{R})$, we see that in $\mathcal{B}(\mathbb{R}),[u] \neq[v]$ and they both have order 10 .

4.3. For $z \in \mathbb{C} \backslash\{0,1\}$, we will still denote by $[z]$ the element it represents in $\mathcal{P}(\mathbb{C})$. By [DS, Sus], We have in $\mathcal{P}(\mathbb{C}), \forall z \in F \backslash\{0,1\}$,

$$
\begin{gathered}
{\left[z^{-1}\right]=-[z] ;} \\
{[1-z]=-[z] .}
\end{gathered}
$$

Let $V$ be a 2-dimensional complex vector space. Given five distinct points $l_{i} \in$ $\mathbb{P}(V), 1 \leq i \leq 5$, we define an element $\mathcal{F}\left(l_{1}, \cdots, l_{5}\right) \in \mathbb{Z}[\mathbb{C} \backslash\{0,1\}]$ as follows:

$$
\mathcal{F}\left(l_{1}, \cdots, l_{5}\right):=\sum_{i=1}^{5}(-1)^{i-1}\left[X\left(l_{1}, \cdots, \hat{l}_{i}, \cdots, l_{5}\right)\right]
$$


where $\hat{l}_{i}$ means that $l_{i}$ is omitted and $X\left(l_{1}, \cdots, \hat{l_{i}}, \cdots, l_{5}\right)$ is the cross ratio as in Definition 5.1.

We can choose a basis of $V$ and express in the homogeneous coordinates $l_{1}=$ $[1,0], l_{2}=[0,1], l_{3}=[1,1], l_{4}=[x, 1], l_{5}=[y, 1]$. Since the $l_{i}$ are distinct, we have $x, y \in \mathbb{C} \backslash\{0,1\}$ and $x \neq y$. By the Definition 5.1, we get:

$$
\mathcal{F}\left(l_{1}, \cdots, l_{5}\right)=[x]-[y]+\left[\frac{y}{x}\right]-\left[\frac{1-y}{1-x}\right]+\left[\frac{1-y^{-1}}{1-x^{-1}}\right] .
$$

Note that this is different from the 5-term relation $T(x, y)$ as in (4.1.1).

Lemma 4.6. With the notations as above.

(1). We have the following identity in $\mathbb{Z}[\mathbb{C} \backslash\{0,1\}]$ :

$$
\mathcal{F}\left(l_{1}, \cdots, l_{5}\right)=T\left(y^{-1}, x^{-1}\right)+\left([x]+\left[x^{-1}\right]\right)-\left([y]+\left[y^{-1}\right]\right) .
$$

(2). In $\mathcal{P}(\mathbb{C}),\left[\mathcal{F}\left(l_{1}, \cdots, l_{5}\right)\right]=0$.

Proof. (1). By (4.1.1), we have

$$
T\left(y^{-1}, x^{-1}\right)=\left[y^{-1}\right]-\left[x^{-1}\right]+\left[\frac{y}{x}\right]-\left[\frac{1-y}{1-x}\right]+\left[\frac{1-y^{-1}}{1-x^{-1}}\right] .
$$

Now by Equation (4.3.4), we obtain

$$
\mathcal{F}\left(l_{1}, \cdots, l_{5}\right)=T\left(y^{-1}, x^{-1}\right)+\left([x]+\left[x^{-1}\right]\right)-\left([y]+\left[y^{-1}\right]\right)
$$

(2). By (4.3.1), we know that for any $t \in \mathbb{C} \backslash\{0,1\},[t]+\left[t^{-1}\right]=0$ in $\mathcal{P}(\mathbb{C})$. Hence, it follows from part (1).

4.4. Relations with Algebraic K-theory. Let $F$ be a field. We first recall the definition of Milnor K-groups of $F$ which was introduced in [Mil]. Let $F^{*}=F \backslash\{0\}$ be the multiplicative group of $F$. Let $T\left(F^{*}\right)$ be its graded tensor algebra over $\mathbb{Z}$. That is,

$$
T\left(F^{*}\right)=\bigoplus_{n \geq 0}\left(F^{*}\right)^{\otimes n},
$$

where $\left(F^{*}\right)^{\otimes 0}=\mathbb{Z},\left(F^{*}\right)^{\otimes 1}=F^{*}$, for $n \geq 2,\left(F^{*}\right)^{\otimes n}=F^{*} \otimes_{\mathbb{Z}} \cdots \otimes_{\mathbb{Z}} F^{*}$ ( $n$ factors $)$.

Let $I$ be the homogeneous ideal of $T\left(F^{*}\right)$ generated by the elements of the form $a \otimes(1-a), a \in F \backslash\{0,1\}$.

Definition 4.7. The Milnor ring of $F$ is the graded ring $K_{*}^{M}=T\left(F^{*}\right) / I$. Its degree $n$ part is called the $n$-th Milnor $K$-group of $F$, denoted by $K_{n}^{M}(F)$.

By definition, since the generators of $I$ are of degree 2, we have

$$
K_{0}^{M}(F)=\mathbb{Z}, K_{1}^{M}(F)=F^{*} .
$$

For $n \geq 2$, as abelian group,

$$
K_{n}^{M}(F)=\left(F^{*}\right)^{\otimes n} / I_{n},
$$

where $I_{n}$ is the subgroup generated by the tensors of the form $a_{1} \otimes \cdots \otimes a_{n}$ with $a_{i}+a_{i+1}=0$ for some $i, 1 \leq i \leq n-1$.

The definition of Quillen's algebraic K-groups $K_{*}(F)$ of $F$ is more involved (See [Qui]). They are defined as the homotopy groups of a certain topological space which is closely related to the classifying space of the infinite general linear group $G L(F)$ of $F$. Here $G L(F)$ is the direct limit of $G L(n, F), n \geq 1$ with respect to the homomorphisms $d_{n}: G L(n, F) \rightarrow G L(n+1, F)$, defined by

$$
d_{n}(A)=\left(\begin{array}{cc}
A & 0 \\
0 & 1
\end{array}\right) \text {. }
$$


It is well-known that $K_{1}(F) \cong K_{1}^{M}(F)=F^{*}$ and $K_{2}^{M}(F) \cong K_{2}(F)$. Moreover, there is a natural homomorphism from $K_{n}^{M}(F)$ to $K_{n}(F)$ for each $n$. The cokernel of this map is called the group of indecomposable elements, denoted by $K_{n}^{\text {ind }}(F)$. The first non-trivial case is when $n=3$.

For more information and details about the algebraic K-theory, besides the original papers [Qui, Mil], we recommend the books [Sri, We] and the references therein. In this paper, we will focus on $K_{3}$.

Now for $n=3$ and $F$ an infinite field, we have the following fundamental exact sequence, due to Suslin ([Sus, Theorem 5.2]):

$$
0 \longrightarrow \operatorname{Tor}(\mu(F), \mu(F))^{\sim} \longrightarrow K_{3}^{\text {ind }}(F) \longrightarrow \mathcal{B}(F) \longrightarrow 0,
$$

where $\mu(F)$ is the group of roots of unity in $F$. $\operatorname{Tor}(\mu(F), \mu(F)) \sim$ is the unique nontrivial extension of $\operatorname{Tor}(\mu(F), \mu(F))$ by $\mathbb{Z} / 2$.

When $F=\mathbb{C}, \operatorname{Tor}(\mu(\mathbb{C}), \mu(\mathbb{C}))^{\sim}=\mathbb{Q} / \mathbb{Z}$, we have the exact sequence:

$$
0 \longrightarrow \mathbb{Q} / \mathbb{Z} \longrightarrow K_{3}^{\text {ind }}(\mathbb{C}) \longrightarrow \mathcal{B}(\mathbb{C}) \longrightarrow 0 .
$$

When $F=\mathbb{R}, \operatorname{Tor}(\mu(\mathbb{R}), \mu(\mathbb{R}))^{\sim}=\mathbb{Z} / 4 \mathbb{Z}$, we have the exact sequence

$$
0 \longrightarrow \mathbb{Z} / 4 \mathbb{Z} \longrightarrow K_{3}^{\text {ind }}(\mathbb{R}) \longrightarrow \mathcal{B}(\mathbb{R}) \longrightarrow 0 .
$$

We also know that (See $[\mathrm{Sah}])$

$$
K_{3}^{\text {ind }}(\mathbb{C}) \simeq H_{3}(S L(2, \mathbb{C}), \mathbb{Z}), K_{3}^{\text {ind }}(\mathbb{R}) \simeq H_{3}(S L(2, \mathbb{R}), \mathbb{Z}),
$$

where the group homologies are the homologies of $S L(2, \mathbb{C})$, and $S L(2, \mathbb{R})$ viewed as discrete groups.

Complex conjugation induces an involution on $H_{3}(S L(2, \mathbb{C}), \mathbb{Z})$, which will be denoted by $c$. Set

$$
H_{3}(S L(2, \mathbb{C}), \mathbb{Z})^{+}=\left\{u \in H_{3}(S L(2, \mathbb{C}), \mathbb{Z}) \mid c(u)=u\right\}
$$

and

$$
H_{3}(S L(2, \mathbb{C}), \mathbb{Z})^{-}=\left\{u \in H_{3}(S L(2, \mathbb{C}), \mathbb{Z}) \mid c(u)=-u\right\} .
$$

By [DWS, Sah], the natural homomorphism $g: H_{3}(S L(2, \mathbb{R}), \mathbb{Z}) \rightarrow H_{3}(S L(2, \mathbb{C}), \mathbb{Z})$ is injective and its image is equal to $H_{3}(S L(2, \mathbb{C}), \mathbb{Z})^{+}$.

Let $f: \mathcal{B}(\mathbb{R}) \rightarrow \mathcal{B}(\mathbb{C})$ be the natural homomorphism induced by the inclusion of $\mathbb{R}$ into $\mathbb{C}$. The following proposition is probably known to the experts, but we can't find a proof in the literature. We include a proof here.

Proposition 4.8. The image of $f$ is $\mathcal{B}(\mathbb{C})^{+}$and the kernel of $f$ is the torsion subgroup of $\mathcal{B}(\mathbb{R})$. Hence $\mathcal{B}(\mathbb{R}) /\{$ torsion $\} \cong \mathcal{B}(\mathbb{C})^{+}$, equivalently, $\mathcal{B}(\mathbb{R}) \otimes \mathbb{Q} \cong$ $\mathcal{B}(\mathbb{C})^{+}$.

Proof. By [DS, Sah, Sus], we have the following commutative diagram:

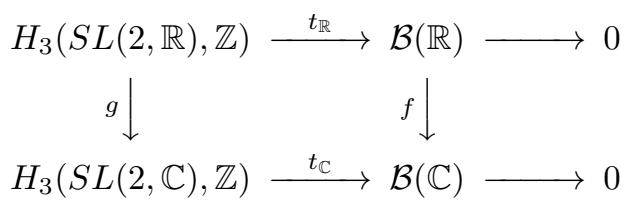

Both kernels of $t_{\mathbb{C}}$ and $t_{\mathbb{R}}$ consist of torsion elements. $g$ is the natural homomorphism induced by the inclusion of $S L(2, \mathbb{R})$ into $S L(2, \mathbb{C})$. The complex conjugation acts both on $H_{3}(S L(2, \mathbb{C}), \mathbb{Z})$ and $\mathcal{B}(\mathbb{C})$. Let $H_{3}(S L(2, \mathbb{C}), \mathbb{Z})^{+}$and $\mathcal{B}(\mathbb{C})^{+}$ be the corresponding subgroups which are invariant under the action. For $a \in$ $H_{3}(S L(2, \mathbb{C}), \mathbb{Z})$ or $a \in \mathcal{B}(\mathbb{C})$, we will denote by $\bar{a}$ its image under the complex 
conjugation. It is known that $t_{\mathbb{C}}$ is compatible with the complex conjugation, i.e. for $a \in H_{3}(S L(2, \mathbb{C}), \mathbb{Z})$, we have

$$
t_{\mathbb{C}}(\bar{a})=\overline{t_{\mathbb{C}}(a)} .
$$

Let's first show that the image of $f$ is $\mathcal{B}(\mathbb{C})^{+}$. By the definition, the image of $f$ is contained in $\mathcal{B}(\mathbb{C})^{+}$. Suppose $a \in \mathcal{B}(\mathbb{C})^{+}$, i.e. $a=\bar{a}$. Since $\mathcal{B}(\mathbb{C})$ is a $\mathbb{Q}$-vector space, there is a unique $b \in \mathcal{B}(\mathbb{C})$ such that $b=\frac{1}{2} a$, i.e. $2 b=a$. Since $a \in \mathcal{B}(\mathbb{C})^{+}$, so is $b$. Since $t_{\mathbb{C}}$ is surjective, there is a $c \in H_{3}(S L(2, \mathbb{C}), \mathbb{Z})$ such that $t_{\mathbb{C}}(c)=b$. Let $x=c+\bar{c}$, then $x \in H_{3}(S L(2, \mathbb{C}), \mathbb{Z})^{+}$and

$$
t_{\mathbb{C}}(x)=t_{\mathbb{C}}(c)+t_{\mathbb{C}}(\bar{c})=b+\bar{b}=2 b=a .
$$

Since $g$ maps $H_{3}(S L(2, \mathbb{R}), \mathbb{Z})$ onto $H_{3}(S L(2, \mathbb{C}), \mathbb{Z})^{+}, \exists y \in H_{3}(S L(2, \mathbb{R}), \mathbb{Z})$ such that $g(y)=x$. By the diagram chasing, we see that $f\left(t_{\mathbb{R}}(y)\right)=a$. Therefore the image of $f$ is $\mathcal{B}(\mathbb{C})^{+}$.

If $a$ is a torsion of $\mathcal{B}(\mathbb{R})$, since $\mathcal{B}(\mathbb{C})$ is torsion-free, we see $f(a)=0$. Conversely suppose $a \in \operatorname{ker} f$. Since $t_{\mathbb{R}}$ is surjective, $\exists b \in H_{3}(S L(2, \mathbb{R}), \mathbb{Z})$ such that $a=t_{\mathbb{R}}(b)$. By the commutative diagram, we obtain $t_{\mathbb{C}}(g(b))=0$. Since ker $t_{\mathbb{C}}$ is torsion, $g(b)$ is a torsion. Since $g$ is injective, $b$ is a torsion. Hence $a=t_{\mathbb{R}}(b)$ is a torsion.

4.5. $H_{3}(\mathrm{SL}(n, \mathbb{C}), \mathbb{Z})$ and $K_{3}(\mathbb{C})$. Let $F$ be a field. The natural imbedding $a_{n}$ : $\mathrm{SL}(n, F) \rightarrow \mathrm{SL}(n+1, F)$ defined by

$$
a_{n}(A)=\left(\begin{array}{cc}
A & 0 \\
0 & 1
\end{array}\right)
$$

induces the natural homomorphism

$$
a(i, n): H_{i}(\mathrm{SL}(n, F), \mathbb{Z}) \rightarrow H_{i}(\mathrm{SL}(n+1, F), \mathbb{Z})
$$

for each $i$ and $n$.

The following proposition was proved by Sah.

Proposition 4.9 (Sah, $[\mathrm{Sah}])$. Let $F=\mathbb{C}$ or $\mathbb{R}$.

(1). $a(3, n): H_{3}(\mathrm{SL}(n, F), \mathbb{Z}) \rightarrow H_{i}(\mathrm{SL}(n+1, F), \mathbb{Z})$ are isomorphisms for $n \geq 3$;

(2). $a(3,2): H_{3}(\mathrm{SL}(2, F), \mathbb{Z}) \rightarrow H_{3}(\mathrm{SL}(3, F), \mathbb{Z})$ is injective.

Moreover, for $F=\mathbb{C}$, we have for each $n \geq 3$,

$$
K_{3}(\mathbb{C}) \simeq H_{3}(\mathrm{SL}(n, \mathbb{C}), \mathbb{Z}),
$$

and

$$
K_{3}(\mathbb{C}) \simeq H_{3}(\mathrm{SL}(2, \mathbb{C}), \mathbb{Z}) \bigoplus K_{3}^{M}(\mathbb{C}) \simeq K_{3}^{\text {ind }}(\mathbb{C}) \bigoplus K_{3}^{M}(\mathbb{C})
$$

\section{Configurations of Flags AND their INVARIANTS}

Three manifolds are triangulated by tetrahedra and the change from one triangulation to another is done through 3-2 or 4-1 moves. For manifolds which are locally a flag manifold it is natural to think of the tetrahedra with vertices given by flags. This will be described in the next section.

In order to obtain an invariant of the manifold, defined in terms of a triangulation and vertices associated to flags, one should prove that it it is independent of the triangulation. In other terms, one should prove invariance under 2-3 or 4-1 moves.

A natural definition involves cross ratios of a configuration of four flags and the invariance property translates into a 5-term relation. We will first recall the definition of the cross ratio of 4 distinct points on a projective line and then review the invariant of four flags. 
5.1. Cross ratio. Let $F$ be a field. Let $V$ be a vector space over $F$. Let $\mathbb{P}(V)$ be the projective space of $V$, that is, $\mathbb{P}(V)$ is the set of 1-dimensional subspaces of $V$.

Suppose $\operatorname{dim}(V)=2$ and we fix a basis of $V$. Given four distinct ordered elements $l_{i}=\left[a_{i}, b_{i}\right] \in \mathbb{P}(V), 1 \leq i \leq 4$, where $\left[a_{i}, b_{i}\right]$ are the corresponding homogeneous coordinates.

Definition 5.1. We define the cross ratio of the four distinct ordered elements $l_{i} \in \mathbb{P}(V)$ by the formula:

$$
X\left(l_{1}, l_{2}, l_{3}, l_{4}\right)=\frac{\left|l_{1}, l_{3}\right|\left|l_{2}, l_{4}\right|}{\left|l_{1}, l_{4}\right|\left|l_{2}, l_{3}\right|},
$$

where $\left|l_{i}, l_{j}\right|$ denotes the determinant of the $2 \times 2$ matrix $\left(\begin{array}{cc}a_{i} & a_{j} \\ b_{i} & b_{j}\end{array}\right)$.

It is well-known that the cross ratio is independent of the choices of the basis of $V$ and it is invariant under the group $\operatorname{PGL}(V)$. In particular, if $l_{i}=\left[x_{i}, 1\right]$, $1 \leq i \leq 4$, then we have

$$
X\left(l_{1}, l_{2}, l_{3}, l_{4}\right)=\frac{\left(x_{1}-x_{3}\right)\left(x_{2}-x_{4}\right)}{\left(x_{1}-x_{4}\right)\left(x_{2}-x_{3}\right)} .
$$

Moreover, it is straightforward to check the following properties of the cross ratio, and we will omit the proof.

Lemma 5.2. (1) Let $c=X\left(l_{1}, l_{2}, l_{3}, l_{4}\right)$, then we have the following identities:

$$
\begin{gathered}
X\left(l_{2}, l_{1}, l_{3}, l_{4}\right)=c^{-1} ; X\left(l_{3}, l_{2}, l_{1}, l_{4}\right)=\frac{1}{1-c^{-1}} ; X\left(l_{4}, l_{2}, l_{3}, l_{1}\right)=1-c ; \\
X\left(l_{2}, l_{3}, l_{1}, l_{4}\right)=\frac{1}{1-c} ; X\left(l_{3}, l_{1}, l_{2}, l_{4}\right)=1-c^{-1} .
\end{gathered}
$$

(2) Let $K_{4}=\{1,(12)(34),(13)(24),(14)(23)\}$ which is a normal subgroup of $S_{4}$. Then $\forall \sigma \in K_{4}$,

$$
X\left(l_{\sigma(1)}, l_{\sigma(2)}, l_{\sigma(3)}, l_{\sigma(4)}\right)=X\left(l_{1}, l_{2}, l_{3}, l_{4}\right) .
$$

Now suppose $\operatorname{dim} V=3$ and consider the projective plane $\mathbb{P}(V)$. For a point $p \in \mathbb{P}(V)$, we define $\mathcal{L}(p)$ to be the set of all the (projective) lines in $\mathbb{P}(V)$ passing through $p$. To avoid any confusion, we will denote by $l_{p}$ the 1-dimensional subspace of $V$ represented by $p$. Clearly $\mathcal{L}(p)$ can be naturally identified with the set of all 2-dimensional subspaces of $V$ which contain $l_{p}$. Let $V^{*}$ be the dual space of $V$. Let $V^{*}(p)=\left\{f \in V^{*} \mid l_{p} \subset \operatorname{ker} f\right\}$. Then $V^{*}(p)$ is a 2-dimensional subspace of $V^{*}$. Moreover, $\mathcal{L}(p)$ can be naturally identified with $\mathbb{P}\left(V^{*}(p)\right)$. Hence it is a projective line. Given 4 distinct ordered lines $l_{i} \in \mathcal{L}(p), 1 \leq i \leq 4$, we will denote their cross ratio by $X\left(p \mid l_{1}, l_{2}, l_{3}, l_{4}\right)$.

The following proposition is well-known (see Figure 1).

Proposition 5.3. Let $p \in \mathbb{P}(V)$ and $L \subset \mathbb{P}(V)$ a (projective) line not containing p. Let $l_{i} \in \mathcal{L}(p), i=1, \cdots, 4$, be 4 distinct lines passing through $p$. Let $y_{i}=L \cap l_{i}$ be the intersection point, $i=1, \cdots, 4$. Then we have

$$
X\left(p \mid l_{1}, l_{2}, l_{3}, l_{4}\right)=X\left(y_{1}, y_{2}, y_{3}, y_{4}\right),
$$

where the cross ratio on the right-hand side is on the line $L$.

The following proposition will be needed in the proof of the main Theorem 5.9 in this section. It relates the cross ratios of four lines passing through a point in a conic and the cross ratio of four other lines passing through a different point but intersecting the conic at the same points. See Figure 2 for the illustration. It is equivalent to Pascal's theorem. 


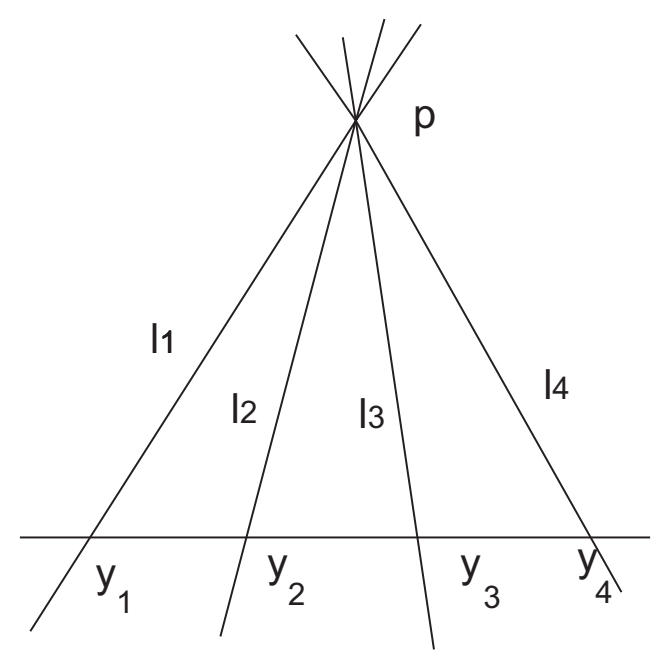

FIGURE 1. The cross ratio of four lines passing through a point $p$

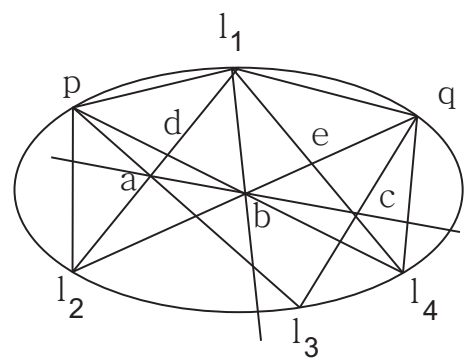

Figure 2. The cross ratio on an irreducible conic

Proposition 5.4. Let $C$ be an irreducible conic in the projective plane $\mathbb{P}(V)$. Fix 4 distinct points $l_{i} \in C, 1 \leq i \leq 4$. For any two points $p \neq q$ on $C$ with $p \neq l_{i}$, $q \neq l_{i}, 1 \leq i \leq 4$, we have the equality:

$$
X\left(p \mid p l_{1}, p l_{2}, p l_{3}, p l_{4}\right)=X\left(q \mid q l_{1}, q l_{2}, q l_{3}, q l_{4}\right),
$$

where for two distinct points $u, v \in \mathbb{P}(V)$, we denote by uv the line passing through them.

Proof. Since $C$ is irreducible, $p l_{1}, p l_{2}, p l_{3}, p l_{4}$ are four distinct lines passing through $p$ and $q l_{1}, q l_{2}, q l_{3}, q l_{4}$ are four distinct lines passing through $q$. Let $a=$ $l_{1} l_{2} \cap p l_{3}, b=q l_{2} \cap p l_{4}, c=l_{1} l_{4} \cap q l_{3}$. By Pascal's theorem (see [Fu, Section 5.6 Corollary 1] for a proof), $a, b, c$ are collinear. Let $d=l_{1} l_{2} \cap p l_{4}, e=l_{1} l_{4} \cap q l_{2}$. By Proposition 5.3, we have

$$
X\left(p \mid l_{1}, l_{2}, l_{3}, l_{4}\right)=X\left(l_{1}, l_{2}, a, d\right)=X\left(b \mid b l_{1}, q l_{2}, b a, p l_{4}\right),
$$

and

$$
X\left(q \mid l_{1}, l_{2}, l_{3}, l_{4}\right)=X\left(l_{1}, e, c, l_{4}\right)=X\left(b \mid b l_{1}, q l_{2}, b c, p l_{4}\right) .
$$

Now since $a, b, c$ are collinear, we have lines $b a=b c$. Therefore,

$$
X\left(b \mid b l_{1}, q l_{2}, b a, p l_{4}\right)=X\left(b \mid b l_{1}, q l_{2}, b c, p l_{4}\right) .
$$

The proposition follows. 
Remark 5.5. See [K, Page 117], where the result is proved by direct computations using a suitable basis and it is used to prove Pascal's theorem.

5.2. In this section, we will take $V$ to be $\mathbb{C}^{3}$. A (complete) flag of $V$ is an ascending sequence of linear subspaces of $V:\{0\} \subset V_{1} \subset V_{2} \subset V$ with $\operatorname{dim} V_{1}=1, \operatorname{dim} V_{2}=$ 2. Let $\operatorname{Flag}(V)$ be the set of all flags of $V$. For a positive integer $m$, we define $\operatorname{Flag}_{m}(V)=\left\{\left(f_{1}, \cdots, f_{m}\right) \mid f_{i} \in \operatorname{Flag}(V), 1 \leq i \leq m\right\}$ to be the set of $m$ ordered flags of $V$. Note that $P G L(3, \mathbb{C})$ acts naturally on $\operatorname{Flag}(V)$, and it acts term-byterm on Flag ${ }_{m}(V)$. The configuration space of $m$ ordered flags of $V$ is defined as the orbit space of $\operatorname{Flag}_{m}(V)$ by the action of $P G L(3, \mathbb{C})$. We will denote it by $\mathcal{C o n f}_{m}$. For $\left(F_{1}, \cdots, F_{m}\right) \in \operatorname{Flag}_{m}(V)$, we will denote by $\left[\left(F_{1}, \cdots, F_{m}\right)\right]$ its orbit in $\mathcal{C o n f}_{m}$.

Since $\operatorname{dim} V=3$ and a hyperplane of $V$ corresponds to the kernel of a linear functional, we obtain

$$
\operatorname{Flag}(V)=\left\{([x],[f]) \in \mathbb{P}(V) \times \mathbb{P}\left(V^{*}\right) \mid f(x)=0\right\} .
$$

Here $[x]$ (resp. $[f]$ ) means the 1-dimensional subspace generated by $x \in V$ (resp. $\left.f \in V^{*}\right) .([x],[f])$ with $f(x)=0$ represents the flag: $\{0\} \subset[x] \subset \operatorname{ker} f \subset V$.

Definition 5.6. Let $\left[\left(\left[x_{1}\right],\left[f_{1}\right]\right), \cdots,\left(\left[x_{m}\right],\left[f_{m}\right]\right)\right] \in \mathcal{C}$ on $f_{m}$. We say it is a generic configuration if $f_{i}\left(x_{j}\right) \neq 0, \forall i \neq j$ and $\left[x_{1}\right], \cdots,\left[x_{m}\right]$ are in generic position in $\mathbb{P}(V)$ (i.e. no three of them lie on a line in $\mathbb{P}(V)$ ). We will denote by $\mathcal{G} c_{m}$ the configuration space of $m$ ordered generic flags.

In the following we will call a generic configuration in $\mathcal{G} c_{4}$ a generic tetrahedron of flags. Now following [BFG] we will introduce the coordinates for them. Let $T=\left(\left[x_{i}\right],\left[f_{i}\right]\right)_{1 \leq i \leq 4}$ be a generic tetrahedron of flags. There are 12 coordinates for the 12 oriented edges of the tetrahedron. The coordinate $z_{i j}(T)$ associated to the edge $i j, 1 \leq i \neq j \leq 4$ is defined as follows. We first choose $k$ and $l$ such that the permutation $(1,2,3,4) \mapsto(i, j, k, l)$ is even. Then the set of all the lines in $\mathbb{P}(V)$ passing through the point $x_{i}$ is a projective line. We have four distinct points on this projective line: the line $\operatorname{ker} f_{i}$ and the three lines $x_{i} x_{l}$ passing through $x_{i}$ and $x_{l}$ for $l \neq i$. We define $z_{i j}(T)$ as the cross ratio of these four points:

$$
z_{i j}(T):=X\left(\left(\operatorname{ker} f_{i}\right),\left(x_{i} x_{j}\right),\left(x_{i} x_{k}\right),\left(x_{i} x_{l}\right)\right) .
$$

By the basic properties of the cross ratio, we see that the edge coordinates leaving a vertex $i$ are related by:

$$
\begin{aligned}
& z_{i k}(T)=\frac{1}{1-z_{i j}(T)} \\
& z_{i l}(T)=1-\frac{1}{z_{i j}(T)}
\end{aligned}
$$

The following proposition is proved in [BFG].

Proposition 5.7. A generic tetrahedron of flags $T \in \mathcal{G} c_{4}$ is uniquely determined by the 4 edge coordinates $\left(z_{12}(T), z_{21}(T), z_{34}(T), z_{43}(T)\right)$ which are in $\mathbb{C} \backslash\{0,1\}$. Hence

$$
\mathcal{G} c_{4} \simeq(\mathbb{C} \backslash\{0,1\})^{4}
$$

Definition 5.8. Let $T \in \mathcal{G} c_{4}$ be a generic tetrahedron with edge coordinates $z_{i j}(T)$, $1 \leq i \neq j \leq 4$. We define a $\mathcal{P}(\mathbb{C})$-valued invariant:

$$
\beta(T)=\left[z_{12}(T)\right]+\left[z_{21}(T)\right]+\left[z_{34}(T)\right]+\left[z_{43}(T)\right] \in \mathcal{P}(\mathbb{C}) .
$$

Let $P=\left(\left[x_{i}\right],\left[f_{i}\right]\right)_{1 \leq i \leq 5} \in \mathcal{G} c_{5}$ be a configuration of of 5 ordered generic flags. Then we have 5 generic tetrahedra $T_{i}$ of flags, $1 \leq i \leq 5$, where $T_{i}$ is the generic tetrahedron obtained from $P$ by deleting the $i$-th flag $\left(\left[x_{i}\right],\left[f_{i}\right]\right)$ of $P$. The order of the 4 flags of $T_{i}$ is induced by the order of $P$. Then we have our main theorem: 


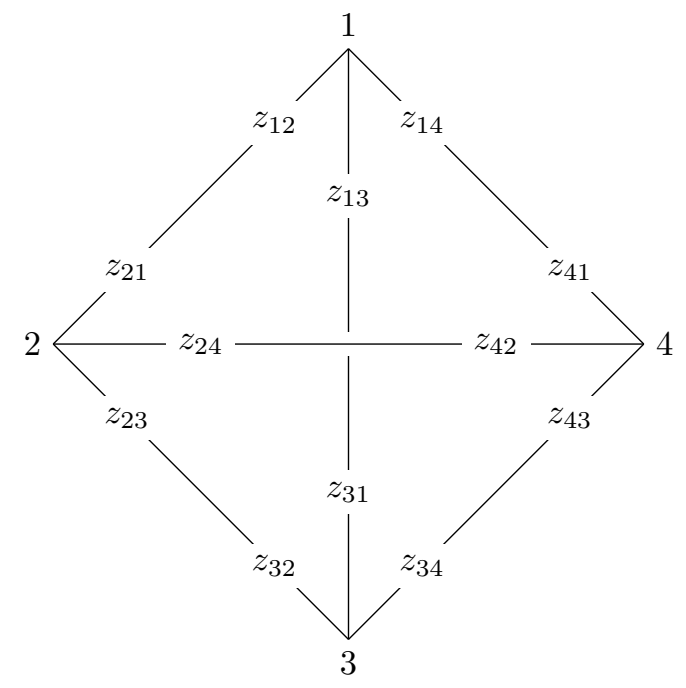

FiguRE 3. The $z$-coordinates.

Theorem 5.9. With the notions as above. We have the following identity in $\mathcal{P}(\mathbb{C})$ :

$$
\sum_{i=1}^{5}(-1)^{i-1} \beta\left(T_{i}\right)=0 .
$$

Proof. By definition, we have

$$
\begin{aligned}
& \beta\left(T_{1}\right)=\left[z_{23}\left(T_{1}\right)\right]+\left[z_{32}\left(T_{1}\right)\right]+\left[z_{45}\left(T_{1}\right)\right]+\left[z_{54}\left(T_{1}\right)\right], \\
& \beta\left(T_{2}\right)=\left[z_{13}\left(T_{2}\right)\right]+\left[z_{31}\left(T_{2}\right)\right]+\left[z_{45}\left(T_{2}\right)\right]+\left[z_{54}\left(T_{2}\right)\right], \\
& \beta\left(T_{3}\right)=\left[z_{12}\left(T_{3}\right)\right]+\left[z_{21}\left(T_{3}\right)\right]+\left[z_{45}\left(T_{3}\right)\right]+\left[z_{54}\left(T_{3}\right)\right], \\
& \beta\left(T_{4}\right)=\left[z_{12}\left(T_{4}\right)\right]+\left[z_{21}\left(T_{4}\right)\right]+\left[z_{35}\left(T_{4}\right)\right]+\left[z_{53}\left(T_{4}\right)\right], \\
& \beta\left(T_{5}\right)=\left[z_{12}\left(T_{5}\right)\right]+\left[z_{21}\left(T_{5}\right)\right]+\left[z_{34}\left(T_{5}\right)\right]+\left[z_{43}\left(T_{5}\right)\right] .
\end{aligned}
$$

To simplify the notations, we will denote by $i j$ the line passing through the points $\left[x_{i}\right]$ and $\left[x_{j}\right]$. Observe that for each point $\left[x_{i}\right] \in \mathbb{P}(V)$ of $P,(1 \leq i \leq 5)$ there are 5 distinct lines passing through $\left[x_{i}\right]$, i.e. $\operatorname{ker} f_{i}$ and lines $i k, 1 \leq k \leq 5, k \neq i$, and the order of the 5 lines is given as follows: for $1 \leq j \leq i-1, l_{j}=i j ; \bar{l}_{i}=\operatorname{ker} f_{i}$; for $i+1 \leq j \leq 5, l_{j}=i j$. Now by Lemma 4.6 and the definition of the invariants $z_{i j}(T)$, we have the following five identities in $\mathcal{P}(\mathbb{C})$, one for each $\left[x_{i}\right]$ :

$$
\begin{aligned}
& I_{1}=[X(12,13,14,15)]-\left[Z_{13}\left(T_{2}\right)\right]+\left[z_{12}\left(T_{3}\right)\right]-\left[z_{12}\left(T_{4}\right)\right]+\left[z_{12}\left(T_{5}\right)\right]=0, \\
& I_{2}=\left[Z_{23}\left(T_{1}\right)\right]-[X(21,23,24,25)]+\left[z_{21}\left(T_{3}\right)\right]-\left[z_{21}\left(T_{4}\right)\right]+\left[z_{21}\left(T_{5}\right)\right]=0, \\
& I_{3}=\left[Z_{32}\left(T_{1}\right)\right]-\left[z_{31}\left(T_{2}\right)\right]+[X(31,32,34,35)]-\left[z_{35}\left(T_{4}\right)\right]+\left[z_{34}\left(T_{5}\right)\right]=0, \\
& I_{4}=\left[Z_{45}\left(T_{1}\right)\right]-\left[z_{45}\left(T_{2}\right)\right]+\left[z_{45}\left(T_{3}\right)\right]-[X(41,42,43,45)]+\left[z_{43}\left(T_{5}\right)\right]=0, \\
& I_{5}=\left[Z_{54}\left(T_{1}\right)\right]-\left[z_{54}\left(T_{2}\right)\right]+\left[z_{54}\left(T_{3}\right)\right]-\left[z_{53}\left(T_{4}\right)\right]+[X(51,52,53,54)]=0 .
\end{aligned}
$$

Hence we have the following identity in $\mathcal{P}(\mathbb{C})$ :

$$
0=\sum_{i=1}^{5} I_{i}=\sum_{i=1}^{5}(-1)^{i-1} \beta\left(T_{i}\right)+R,
$$

where

$$
\begin{aligned}
R & =[X(12,13,14,15)]-[X(21,23,24,25)]+[X(31,32,34,35)] \\
& -[X(41,42,43,45)]+[X(51,52,53,54)] .
\end{aligned}
$$


Since the five point $\left[x_{i}\right]$ are in general position in the projective plane $\mathbb{P}(V)$, there is a unique irreducible conic $C$ passing through them. Pick a point $q \in C$ such that $q \neq\left[x_{i}\right], 1 \leq i \leq 5$. By Proposition 5.4, every cross ratio appearing in $R$ is equal to a cross ratio of lines passing through $q$. That is,

$$
\begin{aligned}
& X(12,13,14,15)=X(q \mid q 2, q 3, q 4, q 5), X(21,23,24,25)=X(q \mid q 1, q 3, q 4, q 5), \\
& X(31,32,34,35)=X(q \mid q 1, q 2, q 4, q 5), X(41,42,43,45)=X(q \mid q 1, q 2, q 3, q 5) \text {, } \\
& X(51,52,53,54)=X(q \mid q 1, q 2, q 3, q 4),
\end{aligned}
$$

where $q i$ means the line passing through the points $q$ and $\left[x_{i}\right]$. Hence we get

$$
R=\sum_{i=1}^{5}(-1)^{i-1} X(q \mid q 1, \cdots, \hat{q} i, \cdots, q 5),
$$

By Lemma 4.6 , we obtain $R=0$ in $\mathcal{P}(\mathbb{C})$. Therefore, by $(5.2 .2)$

$$
\sum_{i=1}^{5}(-1)^{i-1} \beta\left(T_{i}\right)=\sum_{i=1}^{5} I_{i}=0 .
$$

Remark 5.10. For a 3-dimensional manifold $M$ which can be triangulated by finitely many tetrahedra, there are interesting $\mathcal{P}(\mathbb{C})$-valued invariants for representations of $\pi_{1}(M)$ in $P U(2,1)$ and $P G L(3, \mathbb{C})$ ([FW1, BFG]). In order to show that the invariants are well-defined, it is crucial to prove that the invariants are independent of the 2-3 moves of the triangulations of $M$. Theorem 5.9 is equivalent to this. The proof we provide here gives an intuitive and geometric explanation of the calculations carried out in $[\mathrm{Fa}]$.

\section{Representations}

In this section we recall the definition of decorated triangulations of 3-manifolds following [BFG, GGZ15, DGG16]. The definition is related to the case of decorated triangulated surfaces [FG07]. The decoration corresponds to an assignment of a configuration of four complete flags in $\mathbb{C}^{n}$ to each 3 -simplex of the triangulated the 3 -manifold satisfying certain compatibility conditions. The flags in the configuration can be thought as associated to each of the four vertices of a simplex. We will review mainly the case $n=3[\mathrm{BFG}, \mathrm{FKR}]$. Beware that the presentation given here is suitable for $n=3$ and uses the coordinates described in the previous section. But it is not the best set of coordinates for higher dimensional generalizations [GGZ15, DGG16, BBI]. The decorated triangulations give rise to representations of the fundamental group into $\operatorname{PGL}(3, \mathbb{C})$ or $\operatorname{SL}(3, \mathbb{C})$ and the goal is to find, among these representations, those with values in one of the real forms.

6.1. Decorated triangulations of 3-manifolds and representations. We consider an ideal triangulated compact oriented 3 -manifold $M$ with boundary $\partial M$. It means a finite number of oriented 3-simplices $T^{\nu}(1 \leq \nu \leq N)$ and an orientation reversing matching of their faces so that, if one truncates all the simplices at their vertices and identifies the truncated faces, one obtains a manifold homeomorphic to $M$ with $\partial M$ identified to a triangulated 2-surface.

Let $V=\mathbb{C}^{n}$ and $F$ the flag variety of complete flags. We let $G=\operatorname{PGL}(n, \mathbb{C})$ be the projective group acting on the space of flags. 
Definition 6.1. A decoration of an ideal triangulation of a manifold $M$ is a collection of maps from the vertices of each tetrahedron $T^{\nu}$ to the space of configurations $F^{4, \text { gen }} / G$ compatible with the matchings. More precisely, to the 0 -simplex of $T \nu$ one associates a configuration of flags up to the action of $G$ such that two indentified faces are associated with the same point in $F^{3, \text { gen }} / G$.

We impose in the following that the decorations are chosen such that the configurations in $F^{3, \text { gen }}$ have trivial stabilizer. This condition is clearly satisfied for generic configurations as defined in the previous section.

We also assume that the holonomy around the 1-skeleton be trivial. In order to understand this notion of holonomy, let us begin with a simple remark. Consider a tetrahetron $T$, and its decoration which is an element of $F^{4, \text { gen }} / G$. Choosing a lift in $F^{3, g e n}$ for one face determines a lift in $F^{4, g e n}$, because the stabilizer for the face is trivial. Hence, if we fix a lift for one face, we fix the lift for every face. Turning back to the holonomy around the edges: around each edge one chooses an abutted face and consider a lift in $F^{3, \text { gen }}$ of the element in $F^{3, \text { gen }} / G$ associated to the face. Turning around the edge, we fix a lift in $F^{3, \text { gen }}$ for each abutting face. So we construct a sequence of flags corresponding to the vertices (not contained in the edge) of the faces of each of the simplices around the edge. After completing a turn around the edge we suppose that the we recover the same original configuration in $F^{3, \text { gen }}$ (see Figure 4).

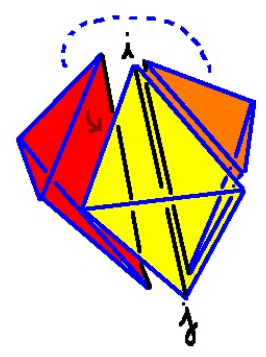

FigURE 4. tetrahedra sharing a common edge

Under this assumption, one obtains a holonomy representation (defined up to conjugation)

$$
\rho: \pi_{1}(M) \longrightarrow \operatorname{PGL}(n, \mathbb{C}) .
$$

Indeed, choose a base face and consider a configuration in $F^{3, \text { gen }}$ associated to it (a lift of the element in $F^{3, \text { gen }} / G$ associated to the face). Any closed path $\gamma$ (based at the chosen face and not intersecting any edge) determines a sequence of configurations in $F^{3, \text { gen }}$ corresponding to the configurations for each successive face crossed by the path. The last face configuration of flags along the path is the image of the reference configuration of flags by a unique element in $G$. This element is called the holonomy along $\gamma$. Clearly, if the holonomy around all edges are trivial, the paths may cross edges without modifying the holonomy and therefore the holonomy representation is well defined on $\pi_{1}(M)$.

From the previous section, we see that a generic decoration gives coordinates $z_{i j}^{\nu}$ for each tetrahedron. One can write algebraic equations which describe the matching between the faces and the triviality of the holonomy around the edges easily. In order to match a face between two tetrahedra $T^{\nu}$ and $T^{\mu}$ one observe 
that the three flags (indexed by $i, j, k$ ) have opposite orientation when viewed with the induced orientation on each tetrahedron. One obtains that the triple ratios, which classify triples up to the action of $\operatorname{PGL}(3, \mathbb{C})$, satisfy

$$
z_{i j k}^{\nu}=1 / z_{i k j}^{\mu}
$$

On the other hand, a simple computation with cross-ratios verifies, taking account of all tetrahedra $T^{\nu}$ having a common edge $i j$, the following conditions

$$
\prod_{\nu} z_{i j}^{\nu}=\prod_{\nu} z_{j i}^{\nu}=1
$$

6.2. Unipotent decorations. It is important to observe that the holonomy along any path $\gamma$ on a boundary component of $M$ corresponding to a vertex of the triangulation preserve the flag associated to this vertex. Indeed, all the faces considered during the computation of the holonomy share this vertex. Hence, the holonomy fixes the flag at this vertex. Up to conjugation the holonomy is therefore contained in a Borel group.

An important class of decorations is obtained by adding the condition that the holonomy of any boundary path is conjugate to a unipotent radical of a Borel subgroup of $G$. In the case of $\operatorname{SL}(n, \mathbb{C})$ or $\operatorname{PGL}(n, \mathbb{C})$, for each boundary component, the boundary holonomy may then be considered to consist of upper triangular matrices with equal entries in the diagonal.

One can compute holonomies in terms of the coordinates $z_{i j}^{\nu}$. Imposing that the boundary holonomy be unipotent can be translated into algebraic equations. One obtains a system of algebraic equations, but due to the the restriction on the coordinates to be generic the solution set is semi-algebraic.

Computations of decorations with complete flags were implemented in $[\mathrm{C}]$ (see also $[\mathrm{FKR}])$. Unipotent decorations were computed for $\operatorname{PGL}(2, \mathbb{C}), \operatorname{PGL}(3, \mathbb{C})$ in many cases and for one manifold, the complement of the figure eight knot, for $\operatorname{PGL}(4, \mathbb{C})$.

\section{3. $G_{0}$-decorations and representations in real forms.}

Definition 6.2. Let $G_{0}$ be a real form of $G$ and $F_{0}$ the closed $G_{0}$-orbit in $F$. A $G_{0}$-decoration of an ideal triangulation of $M$ is a decoration obtained through a family of maps from each tetrahedron to $F_{0}^{4, \text { gen }} / G_{0}$ (here $F_{0}^{4 \text {,gen }}=F^{4 \text {,gen }} \cap F_{0}$ ).

The holonomy representation representation $\rho: \pi_{1}(M) \rightarrow G_{0}$ will be $G_{0}$-valued in this case.

In order to obtain explicit $G_{0}$-decorations we use coordinates for generic flags, excluding certain degenerate configurations. For a given triangulation of a 3-manifold this might result in a loss of certain representations of its fundamental group but passing to a barycentric subdivision one obtains all representations. In generic coordinates, the $G_{0}$-decorations are described as a constructible set given by algebraic equations implying face matchings and trivial holonomy around edges. The condition that the decoration be unipotent is described by additional algebraic equations. We will not reproduce these equations here but refer to references [BFG, GGZ15, DGG16].

A simple condition can be used to distinguish those representations which are in a real form. First of all, if all coordinates $z_{i j}^{\nu}$ are real, the representation is $\mathrm{SL}(3, \mathbb{R})$-valued. For the other non-compact real form we have:

Proposition 6.3 ([BFG]). Let $\left\{T^{\nu}\right\}$ with the data $\nu \rightarrow F^{4, \text { gen }} / G$ be a decorated triangulation and let $z_{i j}^{\nu}$ be the coordinates of each decorated tetrahedron. If, for all $\nu$ and $\{i, j, k, l\}=\{1,2,3,4\}$, we have

$$
z_{i j}^{\nu} z_{j i}^{\nu}=\overline{z_{k l}^{\nu} z_{l k}^{\nu}}
$$


then the holonomy is $\mathrm{PU}(2,1)$-valued.

Unfortunately, computations are difficult and were completed only for triangulations with at most four tetrahedra in the case of $\mathrm{PGL}(3, \mathbb{C})$ or two tetrahedra in the case of $\operatorname{PGL}(4, \mathbb{C})$. The case $\operatorname{PGL}(3, \mathbb{C})$, for manifolds obtained with less than 3 simplices, was treated in [FKR] (see also [CURVE] for a census of manifolds triangulated by four tetrahedra).

6.3.1. The Bloch invariant. Denote an ideal triangulation of a compact manifold $M$ by $K=\bigcup_{\nu} T^{\nu}$ and decoration by the coordinates $z=\left(z_{i j}^{\nu}\right)$.

Definition 6.4. To a decorated triangulation $(K, z)$ with coordinates $z=\left(z_{i j}^{\nu}\right)$ one defines the element

$$
\beta(K, z)=\sum\left[z_{i j}^{\nu}\right] \in \mathcal{P}(\mathbb{C}) .
$$

Changing triangulations through $2-3$ or $1-4$ moves does not change the invariant in $\mathcal{P}(\mathbb{C})$ by Theorem 5.9 .

It was proved in $[\mathrm{BFG}]$ that if the decorated triangulation has unipotent boundary holonomy then the invariant has values in the Bloch group.

In the case of a $G_{0}$-decoration we obtain:

Proposition 6.5. If $(K, z)$ is a $G_{0}$-triangulation then $\beta(K, z) \in \mathcal{P}(\mathbb{C})^{+}$.

The proposition is clear for $G_{0}=\mathrm{SL}(3, \mathbb{R})$. In order to show the proposition for $G_{0}=\mathrm{PU}(2,1)$ it is convenient to use a different set of coordinates. Namely, for a fixed tetrahedron with coordinates $z_{i j}$ define

$$
w_{i j}=z_{i j} z_{j i} \text {. }
$$

Clearly for $G_{0}=\mathrm{PU}(2,1)$ one has $w_{i j}=\bar{w}_{k l}$. Observe then that the triple ratio has a simple formula in those coordinates

$$
-z_{21} z_{31} z_{41}=\frac{1}{w_{34} w_{41} w_{13}}
$$

and similarly for the other triple ratios. One can show that in $\mathcal{P}(\mathbb{C})$ we have (see [FW2]):

$2 \beta(K, z)=\left[w_{12}\right]+\left[w_{21}\right]+\left[w_{34}\right]+\left[w_{43}\right]-\left(\left[w_{12} w_{13} w_{23}\right]+\left[w_{13} w_{34} w_{41}\right]+\left[w_{14} w_{42} w_{21}\right]+\left[w_{24} w_{43} w_{32}\right]\right)+R$

where $R$ is the sum of the following terms

$$
\begin{aligned}
& {\left[\frac{w_{12} w_{14}-w_{12}+1}{w_{12} w_{14}}\right]+\left[\frac{1}{w_{24} w_{12}-w_{24}+1}\right]} \\
& {\left[\frac{w_{12} w_{23}-w_{12}+1}{w_{23} w_{12}}\right]+\left[\frac{1}{w_{31} w_{12}-w_{31}+1}\right]} \\
& {\left[\frac{w_{34} w_{23}-w_{34}+1}{w_{23} w_{34}}\right]+\left[\frac{1}{w_{24} w_{34}-w_{24}+1}\right]} \\
& {\left[\frac{w_{34} w_{14}-w_{34}+1}{w_{34} w_{14}}\right]+\left[\frac{1}{w_{13} w_{34}-w_{13}+1}\right]}
\end{aligned}
$$

The proposition follows from this expression as, except from the boundary term, $\beta(K, z)$ is in $\mathcal{P}(\mathbb{C})^{+}$and we assumed that the boundary terms cancel out. 
6.3.2. Two Questions. Given a decorated ideal triangulation of a 3 -manifold $M$, we can get a $\mathcal{P}(\mathbb{C})$-valued invariant $\beta(\rho)$ for the associated representation $\rho: \pi_{1}(M) \rightarrow$ $\operatorname{PGL}(n, \mathbb{C})$.

Question 1 Let $G_{0}$ be a real form with closed orbit. Suppose the decoration is a $G_{0}$-decoration as in the Definition 6.2 . Is it true that the $\mathcal{P}(\mathbb{C})$-valued invariant $\beta(\rho)$ is contained in the subgroup $\mathcal{P}(\mathbb{C})^{+}$?

For $n=2, G_{0}=P G L(2, \mathbb{R})$ and it is clearly true. For $n=3, G_{0}=P G L(3, \mathbb{R})$ or $P U(2,1)$. In [FW2], we proved that for $P U(2,1)$ the invariant $\beta(\rho)$ lies in $\mathcal{P}(\mathbb{C})^{+}$. Hence it is true for $n=3$.

Question 2 Let $G_{0}$ be a real form with closed orbit. Which elements of $\mathcal{B}(\mathbb{C})^{+}$ and $\mathcal{B}(\mathbb{R})$ can be realized as the invariant $\beta(\rho)$ for $\rho$ coming from a $G_{0}$-decoration?

Note by Proposition 4.8, the difference between $\mathcal{B}(\mathbb{C})^{+}$and $\mathcal{B}(\mathbb{R})$ is the torsion part of $\mathcal{B}(\mathbb{R})$.

For $n=3$, we have some examples which realize some elements in $\mathcal{B}(\mathbb{R})$.

Example 6.6. The $5_{2}$ knot complement. Consider the real root $\gamma$ of the equation $x^{3}-x+1=0$. By [FKR] pages 252-253, the invariant $\beta(\rho)=12[\gamma]$. It is easy to check that $\lambda(\beta(\rho))=12 \gamma \wedge(1-\gamma)=0$. So $\beta(\rho) \in \mathcal{B}(\mathbb{R})$. By the formula (4.2.3), we can numerically compute the value

$$
\frac{12 L\left(\frac{1}{1-\gamma}\right)}{\pi^{2}}
$$

We find it is irrational numerically. This suggests that $\beta(\rho)$ is of infinite order.

Example 6.7. The figure-eight knot's sister manifold. By [BFGKR] page 419, there exist two 1-dimensional components of real solutions. Let $\tau=\frac{1}{2} \pm \frac{1}{2} \sqrt{5}$. Put $X=\tau Y$. The first component is given by

Hence we have the invariant

$$
\left\{\begin{array}{l}
z_{12}=w_{21}=\frac{X+Y}{X-1} ; z_{21}=w_{12}=1+Y \\
z_{34}=w_{43}=\frac{X^{2}+X+Y}{X(X-1)} ; z_{43}=w_{34}=X .
\end{array}\right.
$$

$$
\beta_{1}(Y)=2\left([\tau Y]+[1+Y]+\left[\frac{\tau^{2} Y}{\tau Y-1}\right]+\left[\frac{\tau(Y+1)}{\tau Y-1}\right]\right) .
$$

We can check that $\beta_{1}(Y)=8\left[\tau^{2}\right] \in \mathcal{B}(\mathbb{R})$ which is independent of $Y$.

The second component is given by

$$
\left\{\begin{array}{l}
z_{12}=w_{21}=1+\frac{Y}{X}-\frac{(X+1)(Y+1)}{X^{2}+X-1} \\
z_{21}=w_{12}=\frac{X+Y-1}{Y-1} \\
z_{34}=w_{43}=X+Y \\
z_{43}=w_{34}=\frac{1}{Y}
\end{array}\right.
$$

Hence we have the invariant

$$
\beta_{2}(Y)=2\left(\left[\frac{\tau^{2}(Y-1)}{\tau^{2} Y-1}\right]+\left[\frac{\tau^{2} Y-1}{Y-1}\right]+\left[\tau^{2} Y\right]+\left[\frac{1}{Y}\right]\right) .
$$

We can check that $\beta_{2}(Y)=2\left[\tau^{2}\right] \in \mathcal{B}(\mathbb{R})$.

Let $\tau_{+}=\frac{1}{2}+\frac{1}{2} \sqrt{5}$ and $\tau_{-}=\frac{1}{2}-\frac{1}{2} \sqrt{5}$. By Example 4.5, we know that both $\left[\tau_{+}^{2}\right]$ and $\left[\tau_{-}^{2}\right]$ have order 10 in $\mathcal{B}(\mathbb{R})$. Therefore, both $\beta_{1}(Y)$ and $\beta_{2}(Y)$ are non-trivial torsion elements of $\mathcal{B}(\mathbb{R})$, whose orders are equal to 5 . 
Remark 6.8. The Bloch invariant is related to the Borel invariant: We are given a representation $\rho: \pi_{1}(M) \rightarrow \operatorname{PSL}(3, \mathbb{C})$, of the fundamental group of a compact manifold $M$ which we suppose is a $K\left(\pi_{1}(M), 1\right)$-space. In particular $H^{3}(M, \mathbb{R}) \simeq$ $H^{3}\left(\pi_{1}, \mathbb{R}\right)$. We let $\beta_{c}$ be a generator of the continuous cohomology $H_{c}^{3}(\operatorname{PSL}(3, \mathbb{C}), \mathbb{R}) \simeq$ $\mathbb{R}$ and $\rho^{*}: H_{c}^{3}(\operatorname{PSL}(3, \mathbb{C}), \mathbb{R}) \rightarrow H^{3}\left(\pi_{1}, \mathbb{R}\right)$ be the pull-back of cohomology classes. We define then

$$
B(\rho)=\rho^{*}\left(\beta_{c}\right)([M]),
$$

where $[M]$ is the fundamental class of $M$.

When $M$ has cusps $H_{3}(M, \mathbb{R})=0$ and, in [BBI], the authors were able to represent $\beta_{c}$ by a bounded cocycle defining an element $\beta_{b} \in H_{c b}^{3}(\operatorname{PSL}(3, \mathbb{C}), \mathbb{R})$ where $H_{c b}^{*}$ refers to bounded continuous cohomology. They prove that, for a hyperbolic manifold with cusps, $B(\rho)$ is maximal if and only if $\rho$ is the geometric representation obtained by composition of the geometric representation of the fundamental group into $\mathrm{SL}(2, \mathbb{C})$ with the irreducible representation of $\operatorname{SL}(2, \mathbb{C})$ into $\operatorname{PSL}(n, \mathbb{C})$.

6.3.3. Geometric structures associated to a decorated representation. The computations with coordinates of configurations of flags $G_{0}$-decorations give rise to representations into the real forms $\mathrm{PU}(2,1)$ and $\mathrm{SL}(3, \mathbb{R})$. In both cases, one can, in certain cases, associate $\left(G_{0}, F_{0}\right)$-structures to the 3 -manifold such that its holonomy coincides with the representation. We refer to [Fa, FS] for more details. A basic question is

Question Which 3-manifolds have geometric structures modeled on a real form $G_{0}$ ?

\section{Chern-Simons invariants}

In this section we recall three invariants related to Chern-Simons invariants: one for flag structures, following the Riemannian construction, a second one for representations, via the construction of a flat $G$-bundle and a third one for representations via characteristic classes.

We conclude by the relations - known and less known - relations between these constructions.

7.1. The Chern-Simons invariant of a flag structure. The first invariant is related to the Chern-Simons invariant of a Riemannian manifold. It is defined for flag structures, not necessarily flat ones.

This construction follows the classical Chern-Simons construction in [CS] and, in the case of $\mathrm{CR}$ structures [BE] (see [FV]).

We let $M$ be a real closed 3-manifold equipped with a flag structure. We denote $Y$ the canonical bundle defined above. The second Chern class of the bundle $Y$ with connection form $\pi$ is given by

$$
c_{2}(Y, \pi)=\frac{1}{8 \pi^{2}} \operatorname{Tr}(\Pi \wedge \Pi) .
$$

In the case of the connection form $\pi$ we obtain

$$
\left(\begin{array}{ccc}
0 & -\Phi^{2} & -\frac{1}{4} \Psi \\
0 & 0 & \frac{1}{2} \Phi^{1} \\
0 & 0 & 0
\end{array}\right) \wedge\left(\begin{array}{ccc}
0 & -\Phi^{2} & -\frac{1}{4} \Psi \\
0 & 0 & \frac{1}{2} \Phi^{1} \\
0 & 0 & 0
\end{array}\right)=\left(\begin{array}{ccc}
0 & 0 & -\frac{1}{2} \Phi^{1} \wedge \Phi^{2} \\
0 & 0 & 0 \\
0 & 0 & 0
\end{array}\right)
$$

As $\Phi^{1}=Q^{1} \omega \wedge \omega^{2}$ and $\Phi^{2}=Q^{2} \omega \wedge \omega^{1}$ we have $\Pi \wedge \Pi=0$ and

$$
c_{2}(Y, \pi)=0 .
$$


One observes that the form

$$
T C_{2}(\pi)=\frac{1}{8 \pi^{2}}\left(\operatorname{Tr}(\pi \wedge \Pi)+\frac{1}{3} \operatorname{Tr}(\pi \wedge \pi \wedge \pi)\right)=\frac{1}{24 \pi^{2}} \operatorname{Tr}(\pi \wedge \pi \wedge \pi)
$$

is closed and $0=c_{2}(Y, \pi)=d T C_{2}(\pi)$.

Definition 7.1. Suppose that the fiber bundle $Y \rightarrow M$ is trivial and let $s: M \rightarrow Y$ be a section, we define then

$$
\mu=\int_{M} s^{*} T C_{2}(\pi)=\frac{1}{24 \pi^{2}} \int_{M} s^{*} \operatorname{Tr}(\pi \wedge \pi \wedge \pi) .
$$

In principle that integral depends on the section but the following proposition shows that the integrand

$$
s^{*} T C_{2}(\pi)
$$

defines an element in the cohomology which does not depend on the section.

Proposition 7.2. Suppose $s$ and $\tilde{s}$ are two sections. Let $h: M \rightarrow H$ be such that $\tilde{s}=R_{h} \circ s$. Then

$$
\tilde{s}^{*} T C_{2}(\pi)-s^{*} T C_{2}(\pi)=-\frac{1}{8 \pi^{2}} d s^{*} \operatorname{Tr}\left(h^{-1} \pi \wedge d h\right) .
$$

Let $\mu(t)$ be the invariant defined as a function of the a parameter describing the deformation of the structure on a closed manifold $M$ and define $\delta \mu=\frac{d}{d t} \mu(0)$. The following proposition shows that the set of critical points of this invariant contains the flag structures with zero curvature:

Proposition 7.3. $\delta \mu=-\frac{1}{4 \pi^{2}} \int_{M} s^{*} \operatorname{Tr}(\dot{\pi} \wedge \Pi)$.

In particular, $\mu$ is constant on real flag structures associated to a homotopy class of totally real immersions on the complex flag space.

7.2. The Chern-Simons invariant of a representation. When dealing with a geometric structure on a manifold one obtains a holonomy representation. But, even when the structure is not defined and one is given a representation $\rho: \pi_{1}(M) \rightarrow G$ one can construct an invariant through the flat G-bundle associated to $\rho$. It is defined to be

$$
E_{\rho}=\tilde{M} \times{ }_{\rho} G=\tilde{M} \times G /(x, g) \sim\left(h x, \rho\left(h^{-1}\right) g\right) .
$$

Let $\pi: T E_{\rho} \rightarrow \mathfrak{g}$ be the flat connection on $E_{\rho}$ associated to the representation. Define

$$
C S(\pi)=\frac{1}{8 \pi^{2}}\left(\operatorname{Tr}(\pi \wedge d \pi)+\frac{2}{3} \operatorname{Tr}(\pi \wedge \pi \wedge \pi)\right) .
$$

Suppose now that $E_{\rho}$ (that is the case if $G=\operatorname{SL}(n, \mathbb{C})$ ) is trivial and define for each section of the bundle

$$
c s(\rho)=\int_{M} s^{*} C S(\pi) \quad \bmod \mathbb{Z} .
$$

The invariant above does not depend on the section once we verify that the integration of the form $C S(\pi)$ along the fiber is an integer.

Remark 7.4. In the presence of cusps one can also define an invariant ([M]). 
7.3. The Cheeger-Chern-Simons invariant of a representation. CheegerChern-Simons classes arises from characteristic classes of universal bundles. In the case of $\operatorname{PSL}(2, \mathbb{C})$ (mostly related to hyperbolic geometry) one obtains the map from the third homology of the group considered as a discrete group (see [CS1]):

$$
\hat{c}_{2}: H_{3}(\operatorname{PSL}(2, \mathbb{C}), \mathbb{Z}) \rightarrow \mathbb{C} / \pi^{2} \mathbb{Z}
$$

If $\rho: \pi_{1}(M) \rightarrow \operatorname{PSL}(2, \mathbb{C})$ is a representation of the fundamental group of a $K\left(\pi_{1}(M), 1\right)$-space we have $H_{3}(M, \mathbb{Z})=H_{3}\left(\pi_{1}(M), \mathbb{Z}\right)$ and therefore the fundamental class $[M]$ can be seen inside $H_{3}\left(\pi_{1}(M), \mathbb{Z}\right)$ and its image through $H_{3}\left(\pi_{1}(M), \mathbb{Z}\right) \rightarrow$ $H_{3}(\operatorname{PSL}(2, \mathbb{C}), \mathbb{Z})$ composed with $\hat{c}_{2}$ gives rise to an invariant $\left.i \operatorname{Vol}(\rho)+2 \pi^{2} \operatorname{cs}(\rho)\right)$, where $\operatorname{Vol}(\rho) \in \mathbb{R}$ and $\operatorname{cs}(\rho) \in \mathbb{R} / \mathbb{Z}$. We define $\operatorname{Vol}_{\mathbb{C}}(\rho)=-i \hat{c}_{2}$ to be the complex volume of $\rho$.

In the case of the simply connected group $\mathrm{SL}(2, \mathbb{C})$ one has

$$
\hat{c}_{2}: H_{3}(\mathrm{SL}(2, \mathbb{C}), \mathbb{Z}) \rightarrow \mathbb{C} / 4 \pi^{2} \mathbb{Z} .
$$

If $\rho: \pi_{1}(M) \rightarrow \mathrm{SL}(2, \mathbb{C})$ is a lift of a geometric representation of a closed hyperbolic manifold one obtains that $\hat{c}_{2}(\rho)=i\left(\operatorname{Vol}(M)+i 2 \pi^{2} c s(M)\right)$ as an element in $\mathbb{C} / 2 \pi^{2} \mathbb{Z}$ (theorem $2.8 \mathrm{GTZ}$ ). Here $\operatorname{Vol}(M)$ is the hyperbolic volume of $M$.

Similarly one can define $\hat{c}_{2}: H_{3}(\operatorname{PSL}(n, \mathbb{C}), \mathbb{Z}) \rightarrow \mathbb{C} / \pi^{2} \mathbb{Z}$ and $\hat{c}_{2}: H_{3}(\operatorname{SL}(n, \mathbb{C}), \mathbb{Z}) \rightarrow$ $\mathbb{C} / 4 \pi^{2} \mathbb{Z}$. and the corresponding complex volume invariants of representations $\rho$ : $\pi_{1}(M) \rightarrow \operatorname{PSL}(n, \mathbb{C})$ and $\rho: \pi_{1}(M) \rightarrow \operatorname{SL}(n, \mathbb{C})$ respectively.

An explicit description of the map $\hat{c}_{2}$ is based on the dilogarithm as a function on the Bloch group (See [D, N, BFG, GTZ]).

7.4. Equalities between invariants. As for the relation between the last two Chern-Simmons invariants for representations, one can prove that $c s(\rho)$ coincides with the Chern-Simons invariant defined above in the case the associated PSL $(2, \mathbb{C})$ bundle is trivial. Observe, though, that as $2 \pi^{2} \operatorname{cs}(\rho) \in \mathbb{C} / \mathbb{Z}$ the complex volume misses some information carried by $c s(M)$.

The relation to the first invariant is less known. In general, given a representation, one does not know whether it is the holonomy of a flat structure. However, if this is the case, one can obtain a relation between the invariants. The proof of the following proposition is as in $([\mathrm{Kh}])$ for the case of a spherical CR structure.

Proposition 7.5. Let $M$ be a 3-manifold with a flat flag structure with canonical bundle $Y \rightarrow M$ which we suppose trivial. Denote the holonomy map of the flat structure $\rho: \pi_{1}(M) \rightarrow G$. Then

$$
\mu(M)=-c s(\rho) \quad \bmod \mathbb{Z} .
$$

7.5. $G_{0}$-representations and Chern-Simmons. Consider a representation of the fundamental group of a triangulated manifold $M$ to $\operatorname{PGL}(3, \mathbb{C})$, associated to a decoration.

The real volume can be computed from the coordinates $z_{i j}$ through the Bloch invariant of the decoration, as the sum of the Bloch-Wigner dilogarithm [BBI]. A variant of it, involving flattenings, allows to compute the complex volume and hence the Chern-Simmons invariant.

If the representation takes values in a real form $G_{0}$, then one can prove that the real volume is $0[\mathrm{BFG}]$ but it is not clear what happens to the Chern-Simmons invariant in this case.

Through the database curve [CURVE], one can look at values of the complex volume of unipotent decoration. An observation that can be done is the following one. Each unipotent decoration takes values in a number field, hence gives rise to a family of Galois conjugates. One can check experimentally that, if all the Galois conjugates are inside one real form then the Chern-Simmons invariant $\operatorname{cs}(\rho)$ 
is a simple rationnal number - or equivalently, the complex volume is a rationnal multiple of $2 i \pi^{2}$.

This question raises several interesting questions largely unstudied at this point, related to Galois actions on $\mathcal{B}(\mathbb{C})$.

\section{REFERENCES}

[BFG] N. Bergeron, E. Falbel, A. Guilloux; Tetrahedra of flags, volume and homology of SL(3). Geom. Topol. 18 (2014), no. 4, 1911-1971.

[BFGKR] N. Bergeron, E. Falbel, A. Guilloux, P.-V. Koseleff, F. Rouillier; Local Rigidity for PGL(3,C)-Representations of 3-Manifold Groups. Experimental Mathematics, 22(4):410420, 2013.

[B1] S. Bloch; Higher regulators, algebraic $K$-theory, and zeta functions of elliptic curves. CRM Monograph Series, 11. American Mathematical Society, Providence, RI, 2000.

[BBI] M. Bucher, M. Burger, A. Iozzi; The bounded Borel class and 3-manifold groups. Duke Math. J. 167 (2018), no. 17, 3129-3169

[BE] D. Burns, C. L. Epstein; A global invariant for three-dimensional CR-manifolds, Invent. Math. 92 (1988), no. 2, p. 333-348.

[BS] D. Burns, S. Shnider; Spherical Hypersurfaces in Complex Manifolds. Invent. Math. 33 (1976), 223-246

[C24] E. Cartan; Sur les variétés à connexion projective. Bull. Soc. Math. France 52 (1924), 205-241.

[C] E. Cartan; Sur le groupe de la géométrie hypersphérique, Comm. Math. Helv. 4 (1932), 158-171.

[Ca] A. Casella; Branched Cauchy-Riemann Structures on Once-Punctured Torus Bundles. arXiv:1902.03662

[CS1] J. Cheeger, J. Simons; Differential characters and geometric invariants. Geometry and topology (College Park, Md., 1983/84), 50-80, Lecture Notes in Math., 1167, Springer, Berlin, 1985.

[CM] Chern, S. S. , Moser, J.; Real Hypersurfaces in Complex Manifolds. Acta Math. 133 (1974) 219-271.

[CS] S. S.Chern, J. Simons; Characteristic forms and geometric invariants. Ann. of Math. (2) 99 (1974), 48-69.

[CURVE] http://curve.unhyperbolic.org/

[DGG16] T. Dimofte, M. Gabella, and A. B. Goncharov, K-decompositions and 3d gauge theories, J. High Energy Phys. (2016), no. 11, 151.

[D] J. L. Dupont; The dilogarithm as a characteristic class for flat bundles. J. Pure Appl. Algebra, 44 (1987), 137-164.

[DS] J. L. Dupont, C. H. Sah; Scissors congruences. II. J. Pure Appl. Algebra 25 (1982), no. 2, $159-195$.

[DWS] J. L. Dupont, W. Parry, C.H. Sah; Homology of classical Lie groups made discrete, II, $H_{2}$, $H_{3}$, and relations with scissors congruences, J. Algebra 113 (1988) 215-260.

[Fa] E. Falbel; A volume function for Spherical CR Tetrahedra. Quarterly Journal of Mathematics 62 (2011), no. 2, 397-415.

[FST15] E. Falbel, R. Santos Thebaldi; A flag structure on a cusped hyperbolic 3-manifold, Pacific J. Math. 278 (2015), no.1, 51-78. MR 3404666

[FW1] E. Falbel, Q. Wang ; A combinatorial invariant for Spherical CR structures. Asian J. Math. 17 (2013), no. 3, 391-422.

[FW2] E. Falbel, Q. Wang ; Duality and invariants of representations of fundamental groups of 3-manifolds into PGL(3,C). J. London Math. Soc. (2) 95 (2017) 1-22.

[FKR] E. Falbel, P.-V. Koseleff, F. Rouillier; Representations of fundamental groups of 3manifolds into PGL(3,C):exact computations in low complexity. Geom Dedicata (2015) 177: $229-255$

[FV] E. Falbel, J. M. Veloso; Flag structures on real 3-manifolds. https://arxiv.org/abs/1804.11096.

[FG07] V. V. Fock, A. B. Goncharov, Moduli spaces of convex projective structures on surfaces, Adv. Math. 208 (2007), no. 1, 249-273. MR 2304317

[FS] E. Frenkel, A. Szenes; Crystal bases, dilogarithm identities, and torsion in algebraic Ktheory. J. Amer. Math. Soc. 8 (1995), no. 3, 629-664.

[Fu] W. Fulton; Algebraic curves. An introduction to algebraic geometry. Notes written with the collaboration of Richard Weiss. Mathematics Lecture Notes Series. W. A. Benjamin, Inc., New York-Amsterdam, 1969. 
[GTZ] S. Garoufalidis, D. Thurston, C. Zickert, The complex volume of SL(n,C)-representations of 3-manifolds. Duke Math. J. 164 (2015), no. 11, 2099-2160.

[GGZ15] S. Garoufalidis, M. Goerner, and C. K. Zickert, Gluing equations for PGL $(n, \mathbb{C})-$ representations of 3-manifolds, Algebr. Geom. Topol. 15 (2015), no. 1, 565-622.

[G] A. Guilloux; Deformation of hyperbolic manifolds in PGL(n,C) and discreteness of the peripheral representations. Proc. Amer. Math. Soc. 143 (2015), no. 5, 2215Ñ $1 \S 72226$.

[IL] T. A. Ivey, J. M. Landsberg; Cartan for beginners: differential geometry via moving frames and exterior differential systems. Graduate Studies in Mathematics, 61. American Mathematical Society, Providence, RI, 2003.

[J] H. Jacobowitz; An Introduction to CR Structures. vol. 32, Mathematical Surveys and Monographs, American Mathematical Society, Providence, Rhode Island, 1990.

[K] I. Kaplansky; Linear algebra and geometry. A second course. Reprint of the 1974 revised edition. Dover Publications, 2003.

[Kh] V. T. Khoi; On the Burns-Epstein invariants of spherical CR 3-manifolds. Ann. Inst. Fourier (Grenoble) 61 (2011), no. 2, 775-797.

[Kir] N. A. Kirillov; Dilogarithm identities. Progr. Theoret. Phys. Suppl. No. 118 (1995), 61-142.

[Lic] S. Lichtenbaum; Groups related to scissors-congruence groups. Algebraic K-theory and algebraic number theory (Honolulu, HI, 1987), 151-157, Contemp. Math., 83, Amer. Math. Soc., Providence, RI, 1989 .

[M] G. R. Meyerhoff; THE CHERN-SIMONS INVARIANT FOR HYPERBOLIC 3MANIFOLDS. Thesis (Ph.D.)Princeton University. 1981.

[Mil] J. Milnor; Algebraic K-theory and quadratic forms. Invent. Math. 9 1969/1970 318-344.

[N] W. D. Neumann ; Extended Bloch group and the Cheeger-Chern-Simons class. Geom. Topol. 8, (2004).

[PS] W. Parry, C.H. Sah; Third homology of SL(2,R) made discrete. J. Pure Appl. Algebra 30, 181-209 (1983)

[Qui] D. Quillen; Higher algebraic K-theory. I. Algebraic K-theory, I: Higher K-theories, pp. 85-147. Lecture Notes in Math., Vol. 341, Springer, Berlin 1973.

[Ro] L.J. Rogers; On function sum theorem connected with the series $\sum_{1}^{\infty} \frac{x^{n}}{n^{2}}$, Proc. London Math. Soc. 4, 169-189 (1907)

[Sah] C. H. Sah, Homology of classical Lie groups made discrete. III. J. Pure Appl. Algebra 56 (1989),no. 3, 269-312.

[Sri] V. Srinivas; Algebraic K-theory. Progress in Mathematics, 90. Birkh?user Boston, Inc., Boston, MA, 1991.

[Sus] A. A. Suslin ; $K_{3}$ of a field and the Bloch group. Proc. of the Steklov Institute of Math. Issue 4 (1991), 217-238.

[Ta] N. Tanaka; On the pseudo-conformal geometry of hypersurfaces of the space of $\mathrm{n}$ complex variables. J. Math. Soc. Japan $141962397 \tilde{N} 1 \S 7429$

[T] W. Thurston; The geometry and topology of 3-manifolds. Lecture notes 1979.

[Ti] J. Tits; Sur certaines classes d'espaces homogènes de groupes de Lie. Acad. Roy. Belg. Cl. Sci. Mém. Coll. in 8? 29 (1955), no. 3.

[Wa] H.-C. Wang; Closed manifolds with homogeneous complex structure. Amer. J. Math. 76 (1954), 1-32.

[We] C. Weibel; The K-book. An introduction to algebraic K-theory. Graduate Studies in Mathematics, 145. American Mathematical Society, Providence, RI, 2013.

[Wol69] J. A. Wolf; The action of a real semisimple group on a complex flag manifold. I. Orbit structure and holomorphic arc components, Bull. Amer. Math. Soc. 75 (1969), 1121-1237.

[Za] D. Zagier; The dilogarithm function. Frontiers in number theory, physics, and geometry. II, 3-65, Springer, Berlin, 2007.

Institut de Mathématiques de Jussieu, Université Pierre et Marie Curie, 4 place Jussieu, F-75252 Paris, France INRIA, Ouragan, Paris-Rocquencourt

School of Mathematical Sciences, Fudan University, Shanghai 200433, China

E-mail address: qxwang@fudan.edu.cn 